\title{
Quantitative stability for the Brunn-Minkowski inequality
}

\author{
Alessio Figalli* and David Jerison ${ }^{\dagger}$
}

\begin{abstract}
We prove a quantitative stability result for the Brunn-Minkowski inequality: if $|A|=|B|=1$, $t \in[\tau, 1-\tau]$ with $\tau>0$, and $|t A+(1-t) B|^{1 / n} \leq 1+\delta$ for some small $\delta$, then, up to a translation, both $A$ and $B$ are quantitatively close (in terms of $\delta$ ) to a convex set $\mathcal{K}$.
\end{abstract}

\section{Introduction}

Given two sets $A, B \subset \mathbb{R}^{n}$, and $c>0$, we define the set sum and scalar multiple by

$$
A+B:=\{a+b: a \in A, b \in B\}, \quad c A:=\{c a: a \in A\}
$$

Let $|E|$ denote the Lebesgue measure of a set $E$ (if $E$ is not measurable, $|E|$ denotes the outer Lebesgue measure of $E$ ). The Brunn-Minkowski inequality states that, given $A, B \subset \mathbb{R}^{n}$ nonempty measurable sets,

$$
|A+B|^{1 / n} \geq|A|^{1 / n}+|B|^{1 / n} .
$$

In addition, if $|A|,|B|>0$, then equality holds if and only if there exist a convex set $\mathcal{K} \subset \mathbb{R}^{n}$, $\lambda_{1}, \lambda_{1}>0$, and $v_{1}, v_{2} \in \mathbb{R}^{n}$, such that

$$
\lambda_{1} A+v_{1} \subset \mathcal{K}, \quad \lambda_{2} B+v_{2} \subset \mathcal{K}, \quad\left|\mathcal{K} \backslash\left(\lambda_{1} A+v_{1}\right)\right|=\left|\mathcal{K} \backslash\left(\lambda_{2} B+v_{2}\right)\right|=0 .
$$

Our aim is to investigate the stability of such a statement.

When $n=1$, the following sharp stability result holds as a consequence of classical theorems in additive combinatorics (an elementary proof of this result can be given using Kemperman's theorem [C3, C4]):

Theorem 1.1. Let $A, B \subset \mathbb{R}$ be measurable sets. If $|A+B|<|A|+|B|+\delta$ for some $\delta \leq$ $\min \{|A|,|B|\}$, then there exist two intervals $I, J \subset \mathbb{R}$ such that $A \subset I, B \subset J,|I \backslash A| \leq \delta$, and $|J \backslash B| \leq \delta$.

${ }^{*}$ The University of Texas at Austin, Mathematics Dept. RLM 8.100, 2515 Speedway Stop C1200, Austin, TX 78712-1202 USA. E-mail address: figalli@math.utexas.edu

${ }^{\dagger}$ Department of Mathematics, Massachusetts Institute of Technology, 77 Massachusetts Ave, Cambridge, MA 02139-4307 USA. E-mail address: jerison@math.mit.edu 
Concerning the higher dimensional case, in $\mathrm{C} 1, \mathrm{C} 2$. M. Christ proved a qualitative stability result for (1.2), namely, if $|A+B|^{1 / n}$ is close to $|A|^{1 / n}+|B|^{1 / n}$ then $A$ and $B$ are close to homothetic convex sets.

On the quantitative side, first V. I. Diskant [D] and then H. Groemer [G] obtained some stability results for convex sets in terms of the Hausdorff distance. More recently, sharp stability results in terms of the $L^{1}$ distance have been obtained by the first author together with F. Maggi and A. Pratelli [FMP1, FMP2]. Since this latter result will play a role in our proofs, we state it in detail.

We begin by noticing that, after dilating $A$ and $B$ appropriately, we can assume $|A|=|B|=1$ while replacing the sum $A+B$ by a convex combination $S:=t A+(1-t) B$. It follows by (1.2) that $|S|=1+\delta$ for some $\delta \geq 0$.

Theorem 1.2. (see [FMP1, FMP2]) There is a computable dimensional constant $C_{0}(n)$ such that if $A, B \subset \mathbb{R}^{n}$ are convex sets satisfying $|A|=|B|=1,|t A+(1-t) B|=1+\delta$ for some $t \in[\tau, 1-\tau]$, then, up to a translation,

$$
|A \Delta B| \leq C_{0}(n) \tau^{-1 / 2 n} \delta^{1 / 2}
$$

(Here and in the sequel, $E \Delta F$ denotes the symmetric difference between two sets $E$ and $F$, that is $E \Delta F=(E \backslash F) \cup(F \backslash E)$.)

Our main theorem here is a quantitative version of Christ's result. His result relies on compactness and, for that reason, does not yield any explicit information about the dependence on the parameter $\delta$. Since our proof is by induction on the dimension, it will be convenient to allow the measures of $|A|$ and $|B|$ not to be exactly equal, but just close in terms of $\delta$. Here is the main result of this paper, which shows that the measure of the difference between the sets $A$ and $B$ and their convex hull is bounded by a power $\delta^{\epsilon}$, confirming a conjecture of Christ C1].

Theorem 1.3. Let $n \geq 2$, let $A, B \subset \mathbb{R}^{n}$ be measurable sets, and define $S:=t A+(1-t) B$ for some $t \in[\tau, 1-\tau], 0<\tau \leq 1 / 2$. There are computable dimensional constants $N_{n}$ and computable functions $M_{n}(\tau), \varepsilon_{n}(\tau)>0$ such that if

$$
|| A|-1|+|| B|-1|+|| S|-1| \leq \delta
$$

for some $\delta \leq e^{-M_{n}(\tau)}$, then there exists a convex set $\mathcal{K} \subset \mathbb{R}^{n}$ such that, up to a translation,

$$
A, B \subset \mathcal{K} \quad \text { and } \quad|\mathcal{K} \backslash A|+|\mathcal{K} \backslash B| \leq \tau^{-N_{n}} \delta^{\varepsilon_{n}(\tau)} .
$$

Explicitly, we may take

$$
M_{n}(\tau)=\frac{2^{3^{n+2}} n^{3^{n}}|\log \tau|^{3^{n}}}{\tau^{3^{n}}}, \quad \varepsilon_{n}(\tau)=\frac{\tau^{3^{n}}}{2^{3^{n+1}} n^{3^{n}}|\log \tau|^{3^{n}}} .
$$

It is interesting to make some comments on the above theorem: first of all, notice that the result holds only under the assumption that $\delta$ is sufficiently small, namely $\delta \leq e^{-M_{n}(\tau)}$. A smallness assumption on $\delta$ is actually necessary, as can be easily seen from the following example:

$$
A=B:=B_{\rho}(0) \cup\left\{2 L e_{1}\right\},
$$


where $L \gg 1, e_{1}$ denotes the first vector of the canonical basis in $\mathbb{R}^{n}$, and $\rho>0$ is chosen so that $\left|B_{\rho}(0)\right|=1$. Then it is easily checked that

$$
\left|\frac{1}{2} A+\frac{1}{2} B\right|=\left|B_{\rho}(0) \cup B_{\rho / 2}\left(L e_{1}\right) \cup\left\{2 L e_{1}\right\}\right|=1+2^{-n},
$$

while $|\operatorname{co}(A)| \approx L$ can be arbitrarily large, hence the result is false unless we assume that $\delta<2^{-n}$.

Concerning the exponent $\varepsilon_{n}(\tau)$, at the moment it is unclear to us whether a dimensional dependency is necessary. It is however worth to point out that there are stability results for functional inequalities where a dimensional dependent exponent is needed (see for instance [BP, Theorem 3.5]), so it would not be completely surprising if in this situation the optimal exponent does depend on $n$. We plan to investigate this very delicate question in future works.

Another important direction to develop would be to understand the analytic counterpart of the Brunn-Minkowski inequality, namely the Prékopa-Leindler inequality. At the moment, some stability estimates are known only in one dimension or for some special class of functions [BB1, BB2], and a general stability result would be an important direction of future investigations.

The paper is structured as follows. In the next section we introduce a few notations and give an outline of the proof along with some commentary on the techniques and ideas. Then, in Section 3 we collect most of the technical results we will use. Since the proofs of some of these technical results are delicate and involved, we postpone them to Section 5. Section 4 is devoted to the proof of Theorem 1.3.

Acknowledgements: AF was partially supported by NSF Grant DMS-1262411. DJ was partially supported by the Bergman Trust and NSF Grant DMS-1069225.

\section{Notation and an outline of the proof}

Let $\mathcal{H}^{k}$ denote $k$-dimensional Hausdorff measure on $\mathbb{R}^{n}$. Denote by $x=(y, s) \in \mathbb{R}^{n-1} \times \mathbb{R}$ a point in $\mathbb{R}^{n}$, and let $\pi: \mathbb{R}^{n} \rightarrow \mathbb{R}^{n-1}$ and $\bar{\pi}: \mathbb{R}^{n} \rightarrow \mathbb{R}$ denote the canonical projections, i.e.,

$$
\pi(y, s):=y \quad \text { and } \quad \bar{\pi}(y, s):=s .
$$

Given a compact set $E \subset \mathbb{R}^{n}, y \in \mathbb{R}^{n-1}$, and $\lambda>0$, we use the notation

$$
\begin{gathered}
E_{y}:=E \cap \pi^{-1}(y) \subset\{y\} \times \mathbb{R}, \quad E(s):=E \cap \bar{\pi}^{-1}(s) \subset \mathbb{R}^{n-1} \times\{s\}, \\
\mathcal{E}(\lambda):=\left\{y \in \mathbb{R}^{n-1}: \mathcal{H}^{1}\left(E_{y}\right)>\lambda\right\} .
\end{gathered}
$$

Following Christ [C2], we consider different symmetrizations.

Definition 2.1. Let $E \subset \mathbb{R}^{n}$ be a compact set. We define the Schwarz symmetrization $E^{*}$ of $E$ as follows. For each $t \in \mathbb{R}$,

- If $\mathcal{H}^{n-1}(E(s))>0$, then $E^{*}(s)$ is the closed disk centered at $0 \in \mathbb{R}^{n-1}$ with the same measure.

- If $\mathcal{H}^{n-1}(E(s))=0$, then $E^{*}(s)$ is empty.

We define the Steiner symmetrization $E^{\star}$ of $E$ so that for each $y \in \mathbb{R}^{n-1}$, the set $E_{y}^{\star}$ is empty if $\mathcal{H}^{1}\left(E_{y}\right)=0$; otherwise it is the closed interval of length $\mathcal{H}^{1}\left(E_{y}\right)$ centered at $0 \in \mathbb{R}$. Finally, we define $E^{\natural}:=\left(E^{\star}\right)^{*}$. 


\section{Outline of the proof of Theorem 1.3}

The proof of Theorem 1.3 is very elaborate, combining the techniques of M. Christ with those developed by the present authors in [FJ] (where we proved Theorem 1.3 in the special case $A=B$ and $t=1 / 2$ ), as well as several new ideas. For that reason, we give detailed description of the argument.

In Section 4.1 we prove the theorem in the special case $A=A^{\natural}$ and $B=B^{\natural}$. In this case we have that

$$
A_{y}=\{y\} \times[-a(y), a(y)] \text { and } \quad B_{y}=\{y\} \times[-b(y), b(y)],
$$

for some functions $a, b: \mathbb{R}^{n-1} \rightarrow \mathbb{R}^{+}$, and it is easy to show that $a$ and $b$ satisfy the "3-point concavity inequality"

$$
t a\left(y^{\prime}\right)+(1-t) b\left(y^{\prime \prime}\right) \leq[t a+(1-t) b](y)+\delta^{1 / 4}
$$

whenever $y^{\prime}, y^{\prime \prime}$, and $y:=t y^{\prime}+(1-t) y^{\prime \prime}$ belong to a large subset $F$ of $\pi(A) \cap \pi(B)$. From this 3 -point inequality and an elementary argument (Remark 4.1) we show that $a$ satisfies the "4-point concavity inequality"

$$
a\left(y_{1}\right)+a\left(y_{2}\right) \leq a\left(y_{12}^{\prime}\right)+a\left(y_{12}^{\prime \prime}\right)+\frac{2}{t} \delta^{1 / 4}
$$

with $y_{12}^{\prime}:=t^{\prime} y_{1}+\left(1-t^{\prime}\right) y_{2}, y_{12}^{\prime \prime}:=t^{\prime \prime} y_{1}+\left(1-t^{\prime \prime}\right) y_{2}, t^{\prime}:=\frac{1}{2-t}, t^{\prime \prime}:=1-t^{\prime}$, provided all four points belong to $F$. (The analogous inequality for $b$ involves a different set of four points.)

Using this inequality and Lemma 3.6, we deduce that $a$ is quantitatively close in $L^{1}$ to a concave function. The proof, in Section 5, of Lemma 3.6, although reminiscent of Step 4 in the proof of [FJ, Theorem 1.2], is delicate and involved.

Once we know that $a$ (and analogously $b$ ) is $L^{1}$-close to a concave function, we deduce that both $A$ and $B$ are $L^{1}$-close to convex sets $K_{A}$ and $K_{B}$ respectively, and we would like to say that these convex sets are nearly the same. This is demonstrated as part of Proposition 3.4, which is proved by first showing that $S$ is close to $t K_{A}+(1-t) K_{B}$, then applying Theorem 1.2 to deduce that $K_{A}$ and $K_{B}$ are almost homothetic, and then constructing a convex set $\mathcal{K}$ close to $A$ and $B$ and containing both of them.

This concludes the proof of Theorem 1.3 in the case $A=A^{\natural}$ and $B=B^{\natural}$.

In Section 4.2 we consider the general case, which we prove in several steps, culminating in induction on dimension.

Step 1. This first step is very close to the argument used by M. Christ in [C2], although our analysis is more elaborate since we have to quantify every estimate.

Given $A, B$, and $S$, as in the theorem, we consider their symmetrizations $A^{\natural}, B^{\natural}$, and $S^{\natural}$, and apply the result from Section 4.1 to deduce that $A^{\natural}$ and $B^{\natural}$ are close to the same convex set. This information combined with Christ's Lemma 3.1 allows us to deduce that functions $y \mapsto \mathcal{H}^{1}\left(A_{y}\right)$ and $y \mapsto \mathcal{H}^{1}\left(B_{y}\right)$ are almost equipartitioned (that is, the measure of their level sets $\mathcal{A}(\lambda)$ and $\mathcal{B}(\lambda)$ are very close). This fact combined with a Fubini argument yields that, for most levels $\lambda, \mathcal{A}(\lambda)$ and $\mathcal{B}(\lambda)$ are almost optimal for the $(n-1)$-dimensional Brunn-Minkowski inequality. Thus, by the inductive step, we can find a level $\bar{\lambda} \sim \delta^{\zeta}(\zeta>0)$ such that we can apply the inductive hypothesis to $\mathcal{A}(\bar{\lambda})$ and $\mathcal{B}(\bar{\lambda})$. Consequently, after removing sets of small measure both from $A$ and $B$ and 
translating in $y$, we deduce that $\pi(A), \pi(B) \subset \mathbb{R}^{n-1}$ are close to the same convex set.

Step 2. This step is elementary: we apply a Fubini argument and Theorem 1.1 to most of the sets $A_{y}$ and $B_{y}$ for $y \in \mathcal{A}(\bar{\lambda}) \cap \mathcal{B}(\bar{\lambda})$ to deduce that they are close to their convex hulls. Note, however, that to apply Fubini and Theorem 1.1 it is crucial that, thanks to Step 1, we found a set in $\mathbb{R}^{n-1}$ onto which both $A$ and $B$ project almost fully. Indeed, in order to say that $\mathcal{H}^{1}\left(A_{y}+B_{y}\right) \geq \mathcal{H}^{1}\left(A_{y}\right)+\mathcal{H}^{1}\left(B_{y}\right)$ it is necessary to know that both $A_{y}$ and $B_{y}$ are nonempty, as otherwise the inequality would be false!

Step 3. The argument here uses several ideas from our previous paper [FJ] to obtain a 3-point concavity inequality as in (2.3) above for the "upper profile" of $A$ and $B$ (and an analogous inequality for the "lower profile"). This inequality allows us to say that the barycenter of $A_{y}$ satisfies the 4-point inequality (2.4) both from above and from below, and from this information we can deduce that, as a function of $y$, the barycenter of $A_{y}$ (resp. $B_{y}$ ) is at bounded distance from a linear function (see Lemma 5.1). It follows that the barycenters of $\bar{S}_{y}$ are a bounded distance from a linear function for a set $\bar{S}$ which is almost of full measure inside $S$. Then a variation of [FJ, Proof of Theorem 1.2, Step 3] allows us to show that, after an affine measure preserving transformation, $\bar{S}$ is universally bounded, that is, bounded in diameter by a constant of the form $C_{n} \tau^{-M_{n}}$ where $C_{n}$ and $M_{n}$ are dimensional constants.

Step 4. By a relatively easy argument we find sets $A^{\sim}$ and $B^{\sim}$ of the form

$$
A^{\sim}=\bigcup_{y \in F}\{y\} \times\left[a^{A}(y), b^{A}(y)\right] \quad B^{\sim}=\bigcup_{y \in F}\{y\} \times\left[a^{B}(y), b^{B}(y)\right]
$$

which are close to $A$ and $B$, respectively, and are universally bounded.

Step 5. This is a crucial step: we want to show that $A^{\sim}$ and $B^{\sim}$ are close to convex sets. As in the case $A=A^{\natural}$ and $B=B^{\natural}$, we would like to apply Lemma 3.6 to deduce that $b^{A}$ and $b^{B}$ (resp. $a^{A}$ and $a^{B}$ ) are $L^{1}$-close to concave (resp. convex) functions.

The main issue is that the hypothesis of the lemma, in addition to asking for boundedness and concavity of $b^{A}$ and $b^{B}$ at most points, also requires that the level sets of $b^{A}$ and $b^{B}$ be close to their convex hulls. To deduce this we wish to show that most slices of $A^{\sim}$ and $B^{\sim}$ are nearly optimal in the Brunn-Minkowski inequality in dimension $n-1$ and invoke the inductive hypothesis. We achieve this by an inductive proof of the Brunn-Minkowski inequality, based on combining the validity of Brunn-Minkowski in dimension $n-1$ with 1-dimensional optimal transport (see Lemma 3.5).

An examination of this proof of the Brunn-Minkowski inequality in the situation near equality shows that if $A$ and $B$ are almost optimal for the Brunn-Minkowski inequality in dimension $n$, then for most levels $s$, the slices $A(s)$ and $B(T(s))$ have comparable $(n-1)$-measure, where $T$ is the 1-dimensional optimal transport map, and this pair of sets is almost optimal for the BrunnMinkowski inequality in dimension $n-1$. In particular, we can apply the inductive hypothesis to deduce that most $(n-1)$-dimensional slices are close to their convex hulls.

This nearly suffices to apply Lemma 3.6. But this lemma asks for control of the superlevel sets of the function $b^{A}$, which a priori may be very different from the slices of $A^{\sim}$. To avoid this issue, 
we simply replace $A^{\sim}$ and $B^{\sim}$ by auxiliary sets $A^{-}$and $B^{-}$which consist of the top profile of $A^{\sim}$ and $B^{\sim}$ with a flat bottom, so that the slices coincide with the superlevel sets of $b^{A}$ and $b^{B}$. We then show that Lemma 3.5 applies to $A^{-}$and $B^{-}$. In this way, we end up proving that $A^{\sim}$ and $B^{\sim}$ are close to convex sets, as desired.

Step 6. Since $A^{\sim}$ and $B^{\sim}$ are close to $A$ and $B$ respectively, we simply apply Proposition 3.4 as before in 4.1 to conclude the proof of the theorem.

Step 7. Tracking down the exponents in the proof, we provide an explicit lower (resp. upper) bound on $\varepsilon_{n}(\tau)$ (resp. $\left.M_{n}(\tau)\right)$.

\section{Technical Results}

In this section we state most of the important lemmas we will need. The first three are due to M. Christ (or are easy corollaries of his results).

It is well-known that both the Schwarz and the Steiner symmetrization preserve the measure of sets, while they decrease the measure of the semi-sum (see for instance [C2, Lemma 2.1]). Also, as shown in [C2, Lemma 2.2], the $\downarrow$-symmetrization preserves the measure of the sets $\mathcal{E}(\lambda)$. We combine these results into one lemma, and refer to [C2, Section 2] for a proof.

Lemma 3.1. Let $A, B \subset \mathbb{R}^{n}$ be compact sets. Then $|A|=\left|A^{*}\right|=\left|A^{\star}\right|=\left|A^{\natural}\right|$, $\left|t A^{*}+(1-t) B^{*}\right| \leq|t A+(1-t) B|, \quad\left|t A^{\star}+(1-t) B^{\star}\right| \leq|t A+(1-t) B|, \quad\left|t A^{\natural}+(1-t) B^{\natural}\right| \leq|t A+(1-t) B|$, and, with the notation in (2.2),

$$
\left|A \backslash \pi^{-1}(\mathcal{A}(\lambda))\right|=\left|A^{\natural} \backslash \pi^{-1}\left(\mathcal{A}^{\natural}(\lambda)\right)\right| \quad \text { and } \quad \mathcal{H}^{n-1}(\mathcal{A}(\lambda))=\mathcal{H}^{n-1}\left(\mathcal{A}^{\natural}(\lambda)\right)
$$

for almost every $\lambda>0$.

Another important fact is that a bound on the measure of $t A+(1-t) B$ in terms of the measures of $A$ and $B$ implies bounds relating the sizes of

$$
\sup _{y} \mathcal{H}^{1}\left(A_{y}\right), \quad \sup _{y} \mathcal{H}^{1}\left(B_{y}\right), \quad \mathcal{H}^{n-1}(\pi(A)), \quad \mathcal{H}^{n-1}(\pi(B)) .
$$

Lemma 3.2. Let $A, B \subset \mathbb{R}^{n}$ be compact sets such that $|A|,|B| \geq 1 / 2$ and $|t A+(1-t) B| \leq 2$ for some $t \in(0,1)$, and set $\tau:=\min \{t, 1-t\}$ There exists a dimensional constant $M>1$ such that

$$
\begin{aligned}
\frac{\sup _{y} \mathcal{H}^{1}\left(A_{y}\right)}{\sup _{y} \mathcal{H}^{1}\left(B_{y}\right)} \in\left(\frac{\tau^{n}}{M}, \frac{M}{\tau^{n}}\right), & \frac{\mathcal{H}^{n-1}(\pi(A))}{\mathcal{H}^{n-1}(\pi(B))} \in\left(\frac{\tau^{n}}{M}, \frac{M}{\tau^{n}}\right), \\
\left(\sup _{y} \mathcal{H}^{1}\left(A_{y}\right)\right) \mathcal{H}^{n-1}(\pi(A)) \in\left(\frac{1}{M}, \frac{M}{\tau^{2 n}}\right), & \left(\sup _{y} \mathcal{H}^{1}\left(B_{y}\right)\right) \mathcal{H}^{n-1}(\pi(B)) \in\left(\frac{1}{M}, \frac{M}{\tau^{2 n}}\right) .
\end{aligned}
$$

and, up a measure preserving affine transformation of the form $(y, s) \mapsto\left(\lambda y, \lambda^{1-n} t\right)$ with $\lambda>0$, we have

$$
\mathcal{H}^{n-1}(\pi(A))+\mathcal{H}^{n-1}(\pi(B))+\sup _{y} \mathcal{H}^{1}\left(A_{y}\right)+\sup _{y} \mathcal{H}^{1}\left(B_{y}\right) \leq \frac{M}{\tau^{2 n}}
$$

In this case, we say that $A$ and $B$ are $(M, \tau)$-normalized. 
Proof. As observed in [C2, Lemma 3.1] and in the discussion immediately after that lemma,

$$
\left(\sup _{y} \mathcal{H}^{1}\left(A_{y}\right)\right) \mathcal{H}^{n-1}(\pi(B)) \leq \frac{|t A+(1-t) B|}{t(1-t)^{n-1}} \leq \frac{2}{\tau^{n}}, \quad\left(\sup _{y} \mathcal{H}^{1}\left(A_{y}\right)\right) \mathcal{H}^{n-1}(\pi(A)) \geq|A| \geq 1 / 2 .
$$

By exchanging the roles of $A$ and $B$, the first part of the lemma follows. To prove the second part, it suffices to choose $\lambda>0$ so that $\lambda^{n-1} \mathcal{H}^{n-1}(\pi(A))=1 / \tau^{n}$.

The third lemma is a result of Christ [C1, Lemma 4.1] showing that $\sup _{s} \mathcal{H}^{n-1}(A(s))$ and $\sup _{s} \mathcal{H}^{n-1}(B(s))$ are close in terms of $\delta$ :

Lemma 3.3. Let $A, B \subset \mathbb{R}^{n}$ be compact sets, define $S:=t A+(1-t) B$ for some $t \in[\tau, 1-\tau]$, and assume that (1.3) holds for some $\delta \leq 1 / 2$. Then there exists a numerical constant $L>0$ such that

$$
\frac{\sup _{s} \mathcal{H}^{n-1}(A(s))}{\sup _{s} \mathcal{H}^{n-1}(B(s))} \in\left(1-L \tau^{-1 / 2} \delta^{1 / 2}, 1+L \tau^{-1 / 2} \delta^{1 / 2}\right)
$$

Proof. Set

$$
\gamma:=\left(\frac{\sup _{s} \mathcal{H}^{n-1}(A(s))}{\sup _{s} \mathcal{H}^{n-1}(B(s))}\right)^{1-t}, \quad \tilde{\gamma}:=\left(\frac{\sup _{s} \mathcal{H}^{n-1}(B(s))}{\sup _{s} \mathcal{H}^{n-1}(A(s))}\right)^{t}
$$

and after possibly exchanging $A$ and $B$, we may assume that $\gamma \leq 1$. By the argument in the proof of [C1, Lemma 4.1] we get

$$
|S| \geq t \gamma^{-1}|A|+(1-t) \tilde{\gamma}^{-1}|B|
$$

so, by (1.3),

$$
\left(t \gamma^{-1}+(1-t) \gamma^{t /(1-t)}\right)-1 \leq 4 \delta .
$$

The function

$$
\gamma \mapsto t \gamma^{-1}+(1-t) \gamma^{t /(1-t)}
$$

is convex for $\gamma \in(0,1]$, attains its minimum at $\gamma=1$, and its second derivative is bounded below by $\tau$. It follows that

$$
4 \delta \geq\left(t \gamma^{-1}+(1-t) \gamma^{t /(1-t)}\right)-1 \geq \frac{\tau}{2}|\gamma-1|^{2},
$$

which proves the result.

There are several other important ingredients in the proof of Theorem 1.3, which are to our knowledge new. Because their proofs are long and involved, we postpone them to Section 5 .

The first of these results shows that if $A$ and $B$ are $L^{1}$-close to convex sets $K_{A}$ and $K_{B}$ respectively, then $A$ and $B$ are close to each other, and we can find a convex set $\mathcal{K}$ which contains both $A$ and $B$ with a good control on the measure. As we shall see, the proof relies primarily on Theorem [1.2,

Proposition 3.4. Let $A, B \subset \mathbb{R}^{n}$ be compact sets, define $S:=t A+(1-t) B$ for some $t \in[\tau, 1-\tau]$, and assume that (1.3) holds. Suppose $A, B \subset B_{R}$, for some $R \leq \tau^{-N_{n}}$ with $N_{n}$ a dimensional constant $N_{n}>1$. Suppose further that we can find a convex sets $K_{A}, K_{B} \subset \mathbb{R}^{n}$ such that

$$
\left|A \Delta K_{A}\right|+\left|B \Delta K_{B}\right| \leq \zeta
$$


for some $\zeta \geq \delta$. Then there exists a dimensional constant $L_{n}>1$ such that after a translation,

$$
|A \Delta B| \leq \tau^{-L_{n}} \zeta^{1 / 2 n}
$$

and there exists a convex set $\mathcal{K}$ containing both $A$ and $B$ such that

$$
|\mathcal{K} \backslash A|+|\mathcal{K} \backslash B| \leq \tau^{-L_{n}} \zeta^{1 / 2 n^{3}} .
$$

Our next result is a consequence of a proof of the Brunn-Minkowski inequality by induction, using horizontal $(n-1)$-dimensional slices. The lemma says that when $A, B$, and $S$ satisfy (1.3), then most of their horizontal slices (chosen at suitable levels) satisfy near equality in the BrunnMinkowski inequality and the ratio of their volumes of the slices is comparable to 1 on a large set.

Lemma 3.5. Given compact sets $A, B \subset \mathbb{R}^{n}$ and $S:=t A+(1-t) B$, and recalling the notation $E(s) \subset \mathbb{R}^{n-1} \times\{s\}$ in (2.1), we define the probability densities on the real line

$$
\rho_{A}(s):=\frac{\mathcal{H}^{n-1}(A(s))}{|A|}, \quad \rho_{B}(s):=\frac{\mathcal{H}^{n-1}(B(s))}{|B|}, \quad \rho_{S}(s):=\frac{\mathcal{H}^{n-1}(S(s))}{|S|} .
$$

Let $T: \mathbb{R} \rightarrow \mathbb{R}$ be the monotone rearrangement sending $\rho_{A}$ onto $\rho_{B}$, that is $T$ is an increasing map such that $T_{\sharp} \rho_{A}=\rho_{B} 1$ Then

$$
|S|-\left(t|A|^{1 / n}+(1-t)|B|^{1 / n}\right)^{n} \geq \int_{\mathbb{R}} e_{n-1}(s)\left(t+(1-t) T^{\prime}(s)\right) d s,
$$

where $T_{t}(s):=t s+(1-t) T(s)$ and

$$
e_{n-1}(s):=\mathcal{H}^{n-1}\left(S\left(T_{t}(s)\right)\right)-\left[t \mathcal{H}^{n-1}(A(s))^{1 /(n-1)}+(1-t) \mathcal{H}^{n-1}(B(T(s)))^{1 /(n-1)}\right]^{n-1} .
$$

Moreover, if $t \in[\tau, 1-\tau]$ and (1.3) holds with $\delta / \tau^{n}$ is sufficiently small, then

$$
\int_{\mathbb{R}}\left|\frac{\rho_{A}(s)}{\rho_{B}(T(s))}-1\right| \rho_{A}(s) d s \leq \frac{C(n)}{\tau^{n / 2}} \delta^{1 / 2} .
$$

Finally, we have a lemma saying that if a function $\psi$ is nearly concave on a large set, and most of its level sets are close to their convex hulls, then it is $L^{1}$-close to a concave function. Here and in the sequel, given a set $E$ we will use $\operatorname{co}(E)$ to denote its convex hull.

${ }^{1} T_{\sharp}$ denotes the push-forward through the map $T$, that is,

$$
T_{\sharp} \rho_{A}=\rho_{B} \quad \Leftrightarrow \quad \int_{E} \rho_{B}(s) d s=\int_{T^{-1}(E)} \rho_{A}(s) d s \quad \forall E \subset \mathbb{R} \text { Borel. }
$$

An explicit formula for $T$ can be given using the distribution functions of $\rho_{A}$ and $\rho_{B}$ : if we define

$$
G_{A}(s):=\int_{-\infty}^{s} \rho_{A}\left(s^{\prime}\right) d s^{\prime}, \quad G_{B}(s):=\int_{-\infty}^{s} \rho_{B}\left(s^{\prime}\right) d s^{\prime}
$$

and we set $G_{B}^{-1}(r):=\inf \left\{s \in \mathbb{R}: G_{A}(s)>t\right\}$, then $T=G_{B}^{-1} \circ G_{A}$. 
Lemma 3.6. Let $0<\tau \leq 1 / 2$ and fix $t^{\prime}$ such that $1 / 2 \leq t^{\prime} \leq 1-\tau / 2$. Let $t^{\prime \prime}=1-t^{\prime}$, and for all $y_{1}$ and $y_{2}$ in $\mathbb{R}^{n-1}$ define

$$
y_{12}^{\prime}:=t^{\prime} y_{1}+t^{\prime \prime} y_{2} ; \quad y_{12}^{\prime \prime}:=t^{\prime \prime} y_{1}+t^{\prime} y_{2} .
$$

Let $\sigma, \varsigma>0, \hat{M} \geq 1, F \subset \mathbb{R}^{n-1}$, and let $\psi: F \rightarrow \mathbb{R}$ be a function satisfying

$$
\begin{gathered}
\psi\left(y_{1}\right)+\psi\left(y_{2}\right) \leq \psi\left(y_{12}^{\prime}\right)+\psi\left(y_{12}^{\prime \prime}\right)+\sigma \quad \forall y_{1}, y_{2}, y_{12}^{\prime}, y_{12}^{\prime \prime} \in F, \\
\Omega:=\operatorname{co}(F), \quad \mathcal{H}^{n-1}(\Omega \backslash F) \leq \varsigma, \\
B_{r} \subset \Omega \subset B_{(n-1) r}, \quad 1 / n<r<n, \\
-\hat{M} \leq \psi(y) \leq \hat{M} \quad \forall y \in F .
\end{gathered}
$$

Also, we assume that there exists a set $H \subset \mathbb{R}$ such that

$$
\int_{H} \mathcal{H}^{n-1}(\operatorname{co}(\{\psi>s\}) \backslash\{\psi>s\}) d s+\int_{\mathbb{R} \backslash H} \mathcal{H}^{n-1}(\{\psi>s\}) d s \leq \varsigma .
$$

Then there exist a concave function $\Psi: \Omega \rightarrow[-2 \hat{M}, 2 \hat{M}]$ and a dimensional constant $L_{n}^{\prime}$ such that

$$
\int_{F}|\Psi(y)-\psi(y)| d y \leq \tau^{-L_{n}^{\prime}} \hat{M}(\sigma+\varsigma)^{\beta_{n, \tau}}
$$

where

$$
\beta_{n, \tau}:=\frac{\tau}{16(n-1)|\log \tau|}
$$

\section{Proof of Theorem 1.3}

As explained in [FJ], by inner approximation 2 it suffices to prove the result when $A, B$ are compact sets. Hence, let $A$ and $B$ be compact sets, define $S:=t A+(1-t) B$ for some $t \in[\tau, 1-\tau]$, and assume that (1.3) holds. We want to prove that there exists a convex set $\mathcal{K}$ such that, up to a translation,

$$
A, B \subset \mathcal{K}, \quad|\mathcal{K} \backslash A|+|\mathcal{K} \backslash B| \leq \tau^{-N_{n}} \delta^{\varepsilon_{n}(\tau)} .
$$

In order to simplify the notation, $C$ will denote a generic constant, which may change from line to line, and that is bounded from above by $\tau^{-N_{n}}$ for some dimensional constant $N_{n}>1$ (recall that by assumption $\tau \leq 1 / 2$ ). We will say that such a constant is universal.

Observe that, since the statement and the conclusions are invariant under measure preserving affine transformations, by Lemma 3.2 we can assume that $A$ and $B$ are $(M, \tau)$-normalized (see (3.1) ).

\footnotetext{
${ }^{2}$ The approximation of $A$ (and analogously for $B$ ) is by a sequence of compact sets $A_{k} \subset A$ such that $\left|A_{k}\right| \rightarrow|A|$ and $\left|\operatorname{co}\left(A_{k}\right)\right| \rightarrow|\operatorname{co}(A)|$. One way to construct such sets is to define $A_{k}:=A_{k}^{\prime} \cup V_{k}$, where $A_{k}^{\prime} \subset A$ are compact sets satisfying $\left|A_{k}^{\prime}\right| \rightarrow|A|$, and $V_{k} \subset V_{k+1} \subset A$ are finite sets satisfying $\left|\operatorname{co}\left(V_{k}\right)\right| \rightarrow|\operatorname{co}(A)|$.
} 


\subsection{The case $A=A^{\natural}$ and $B=B^{\natural}$}

Let $A, B \subset \mathbb{R}^{n}$ be compact sets satisfying $A=A^{\natural}, B=B^{\natural}$. Since $\pi(A(s)) \subset \pi(A(0))=\pi(A)$ and $\pi(B(s)) \subset \pi(B(0))=\pi(B)$ are disks centered at the origin, applying Lemma 3.3 we deduce that

$$
\mathcal{H}^{n-1}(\pi(A) \Delta \pi(B)) \leq C \delta^{1 / 2} .
$$

Hence, if we define

$$
\bar{S}:=\bigcup_{y \in \pi(A) \cap \pi(B)} t A_{y}+(1-t) B_{y},
$$

then $\bar{S}_{y} \subset S_{y}$ for all $y \in \mathbb{R}^{n-1}$. In addition, using (1.3), (3.1), and (4.1), we have

$$
\begin{aligned}
1+\delta & \geq|S|=\int_{\mathbb{R}^{n-1}} \mathcal{H}^{1}\left(S_{y}\right) d y \geq \int_{\pi(A) \cap \pi(B)} \mathcal{H}^{1}\left(S_{y}\right) d y \geq \int_{\pi(A) \cap \pi(B)} \mathcal{H}^{1}\left(\bar{S}_{y}\right) d y \\
& =|\bar{S}| \geq t \int_{\pi(A) \cap \pi(B)} \mathcal{H}^{1}\left(A_{y}\right) d y+(1-t) \int_{\pi(A) \cap \pi(B)} \mathcal{H}^{1}\left(B_{y}\right) d y \\
& \geq \frac{t|A|+(1-t)|B|}{2}-C \mathcal{H}^{n-1}(\pi(A) \Delta \pi(B)) \geq 1-C \delta^{1 / 2}
\end{aligned}
$$

which implies (since $\bar{S} \subset S$ )

$$
|S \backslash \bar{S}| \leq C \delta^{1 / 2}
$$

Also, by Chebyshev's inequality we deduce that there exists a set $F \subset \pi(A) \cap \pi(B)$ such that

$$
\mathcal{H}^{n-1}((\pi(A) \cap \pi(B)) \backslash F) \leq C \delta^{1 / 4}, \quad \mathcal{H}^{1}\left(S_{y} \backslash \bar{S}_{y}\right) \leq \delta^{1 / 4} \quad \forall y \in F .
$$

This implies that, if we write

$$
A_{y}:=\{y\} \times[-a(y), a(y)] \text { and } \quad B_{y}:=\{y\} \times[-b(y), b(y)],
$$

with $a$ and $b$ radial decreasing, then

$$
t a\left(y^{\prime}\right)+(1-t) b\left(y^{\prime \prime}\right) \leq[t a+(1-t) b](y)+\delta^{1 / 4} \quad \forall y=t y^{\prime}+(1-t) y^{\prime \prime}, y, y^{\prime}, y^{\prime \prime} \in F .
$$

We show next that a three-point inequality for two functions $f$ and $g$ implies a four-point inequality for each of $f$ and $g$ separately.

Remark 4.1. Let $F \subset \mathbb{R}^{n-1}$, and $f, g: F \rightarrow \mathbb{R}$ be two bounded Borel functions satisfying

$$
t f\left(y^{\prime}\right)+(1-t) g\left(y^{\prime \prime}\right) \leq[t f+(1-t) g](y)+\sigma \quad \forall y=t y^{\prime}+(1-t) y^{\prime \prime}, y, y^{\prime}, y^{\prime \prime} \in F,
$$

for some $\sigma \geq 0$. Let $t \in[\tau, 1-\tau]$ and define

$$
t^{\prime}:=\frac{1}{2-t}, \quad t^{\prime \prime}:=1-t^{\prime} ; \quad y_{12}^{\prime}:=t^{\prime} y_{1}+\left(1-t^{\prime}\right) y_{2}, \quad y_{12}^{\prime \prime}:=t^{\prime \prime} y_{1}+\left(1-t^{\prime \prime}\right) y_{2}
$$


We claim that

$$
f\left(y_{1}\right)+f\left(y_{2}\right) \leq f\left(y_{12}^{\prime}\right)+f\left(y_{12}^{\prime \prime}\right)+\frac{2}{t} \sigma .
$$

(The analogous statement for $g$ involves replacing $t$ with $1-t$, so gives different values of $t^{\prime}$ and $t^{\prime \prime}$.) Notice that, if $\tau \leq t \leq 1 / 2$, then

$$
1 / 2 \leq t^{\prime} \leq 2 / 3
$$

independent of $\tau$, whereas if $1 / 2 \leq t \leq 1-\tau$, then

$$
2 / 3 \leq t^{\prime} \leq 1-\tau / 2 .
$$

To prove (4.4), note that the definitions above imply

$$
y_{12}^{\prime}=t y_{1}+(1-t) y_{12}^{\prime \prime}, \quad y_{12}^{\prime \prime}=t y_{12}^{\prime}+(1-t) y_{2} .
$$

Hence, assuming that $y_{1}, y_{2}, y_{12}^{\prime}, y_{12}^{\prime \prime} \in F$, we can add together the two inequalities

$$
\begin{aligned}
& t f\left(y_{1}\right)+(1-t) g\left(y_{12}^{\prime \prime}\right) \leq[t f+(1-t) g]\left(y_{12}^{\prime}\right)+\sigma, \\
& t f\left(y_{2}\right)+(1-t) g\left(y_{12}^{\prime}\right) \leq[t f+(1-t) g]\left(y_{12}^{\prime \prime}\right)+\sigma,
\end{aligned}
$$

to get (4.4).

By the remark above and Lemma 3.6 (notice that the level sets of $a$ and $b$ are both disks, so (3.10) holds with $\varsigma=0$ ), we obtain that both functions $a$ and $b$ are $L^{1}$-close to concave functions $\Psi_{A}$ and $\Psi_{B}$, both defined on $\pi(A) \cap \pi(B)$. Hence, if we define the convex sets

$$
\begin{aligned}
& K_{A}:=\left\{(y, s) \in \mathbb{R}^{n}: y \in \pi(A) \cap \pi(B),-\Psi_{A}(y) \leq s \leq \Psi_{A}(y)\right\}, \\
& K_{B}:=\left\{(y, s) \in \mathbb{R}^{n}: y \in \pi(A) \cap \pi(B),-\Psi_{B}(y) \leq s \leq \Psi_{B}(y)\right\},
\end{aligned}
$$

we deduce that

$$
\left|A \Delta K_{A}\right|+\left|B \Delta K_{B}\right| \leq C \delta^{\beta_{n, \tau} / 4} .
$$

Hence, it follows from Proposition 3.4 that, up to a translation, there exists a convex set $K$ such that $A \cup B \subset K$ and

$$
|A \Delta B| \leq C \delta^{\beta_{n, \tau} / 8 n}, \quad|K \backslash A|+|K \backslash B| \leq C \delta^{\beta_{n, \tau} / 8 n^{3}} .
$$

Notice that, because $A=A^{\natural}$ and $B=B^{\natural}$, it is easy to check that the above properties still hold with $K^{\natural}$ in place of $K$. Hence, in this case, without loss of generality one can assume that $K=K^{\natural}$.

\subsection{The general case}

Since the result is true when $n=1$ (by Theorem 1.1), we assume that we already proved Theorem 1.3 through $n-1$, and we want to show its validity for $n$. 
Step 1: There exist a dimensional constant $\zeta>0$ and $\bar{\lambda} \sim \delta^{\zeta}$ such that the inductive hypothesis applies to $\mathcal{A}(\bar{\lambda})$ and $\mathcal{B}(\bar{\lambda})$.

Let $A^{\natural}$ and $B^{\natural}$ be as in Definition 2.1. Thanks to Lemma 3.1, $A^{\natural}$ and $B^{\natural}$ still satisfy (1.3), so we can apply the result proved in Section 4.1 above to get (see (4.7))

$$
\int_{\mathbb{R}^{n-1}}\left|\mathcal{H}^{1}\left(A_{y}^{\natural}\right)-\mathcal{H}^{1}\left(B_{y}^{\natural}\right)\right| d y \leq \int_{\mathbb{R}^{n-1}}\left|\mathcal{H}^{1}\left(A_{y}^{\natural} \Delta B_{y}^{\natural}\right)\right| d y=\left|A^{\natural} \Delta B^{\natural}\right| \leq C \delta^{\bar{\alpha}}
$$

and

$$
K \supset A^{\natural} \cup B^{\natural}, \quad\left|K \backslash A^{\natural}\right|+\left|K \backslash B^{\natural}\right| \leq C \delta^{\bar{\alpha} / n^{2}}
$$

for some convex set $K=K^{\natural}$, where

$$
\bar{\alpha}:=\frac{\beta_{n, \tau}}{8 n} .
$$

In addition, because $A$ and $B$ are $(M, \tau)$-normalized (see (3.1)), so are $A^{\natural}$ and $B^{\natural}$, and by (4.9) we deduce that there exists a universal constant $R>0$ such that

$$
K \subset B_{R}
$$

Also, by (4.8) and Chebyshev's inequality we obtain that, up to a set of measure $\leq C \delta^{\bar{\alpha} / 2}$,

$$
\left|\mathcal{H}^{1}\left(A_{y}^{\natural}\right)-\mathcal{H}^{1}\left(B_{y}^{\natural}\right)\right| \leq \delta^{\bar{\alpha} / 2} .
$$

Thus, recalling Lemma 3.1, for almost every $\lambda>0$

$$
\mathcal{H}^{n-1}(\mathcal{A}(\lambda))=\mathcal{H}^{n-1}\left(\mathcal{A}^{\natural}(\lambda)\right) \leq \mathcal{H}^{n-1}\left(\mathcal{B}^{\natural}\left(\lambda-\delta^{\bar{\alpha} / 2}\right)\right)+C \delta^{\bar{\alpha} / 2}=\mathcal{H}^{n-1}\left(\mathcal{B}\left(\lambda-\delta^{\bar{\alpha} / 2}\right)\right)+C \delta^{\bar{\alpha} / 2} .
$$

Since, by (3.1),

$$
\int_{0}^{\tau^{-2 n} M}\left(\mathcal{H}^{n-1}(\mathcal{B}(\lambda))-\mathcal{H}^{n-1}\left(\mathcal{B}\left(\lambda+\delta^{\bar{\alpha} / 2}\right)\right)\right) d \lambda=\int_{0}^{\delta^{\bar{\alpha} / 2}} \mathcal{H}^{n-1}(\mathcal{B}(\lambda)) d \lambda \leq C \delta^{\bar{\alpha} / 2},
$$

by Chebyshev's inequality we deduce that

$$
\mathcal{H}^{n-1}(\mathcal{A}(\lambda)) \leq \mathcal{H}^{n-1}(\mathcal{B}(\lambda))+C \delta^{\bar{\alpha} / 4}
$$

for all $\lambda$ outside a set of measure $\delta^{\bar{\alpha} / 4}$. Exchanging the roles of $A$ and $B$ we obtain that there exists a set $G \subset\left[0, \tau^{-2 n} M\right]$ such that

$$
\mathcal{H}^{1}(G) \leq C \delta^{\bar{\alpha} / 4}, \quad\left|\mathcal{H}^{n-1}(\mathcal{A}(\lambda))-\mathcal{H}^{n-1}(\mathcal{B}(\lambda))\right| \leq C \delta^{\bar{\alpha} / 4} \quad \forall \lambda \in[0, \infty] \backslash G .
$$

Using the elementary inequality

$$
(t a+(1-t) b)^{n-1} \geq t a^{n-1}+(1-t) b^{n-1}-C|a-b|^{2} \quad \forall 0 \leq a, b \leq \frac{M}{\tau^{2 n}},
$$

and replacing $a$ and $b$ with $a^{1 /(n-1)}$ and $b^{1 /(n-1)}$, respectively, we get

$$
\left(t a^{1 /(n-1)}+(1-t) b^{1 /(n-1)}\right)^{n-1} \geq t a+(1-t) b-C|a-b|^{2 /(n-1)} \quad \forall 0 \leq a, b \leq \frac{M}{\tau^{2 n}}
$$


(notice that $\left|a^{1 /(n-1)}-b^{1 /(n-1)}\right| \leq|a-b|^{1 /(n-1)}$ ). Finally, it is easy to check that

$$
t \mathcal{A}(\lambda)+(1-t) \mathcal{B}(\lambda) \subset \mathcal{S}(\lambda) \quad \forall \lambda>0 .
$$

Hence, by the Brunn-Minkowski inequality (1.2) applied to $\mathcal{A}(\lambda)$ and $\mathcal{B}(\lambda)$, using (1.3), (3.1), (4.13), and (4.12), we get

$$
\begin{aligned}
1+\delta \geq|S| & =\int_{0}^{\tau^{-2 n} M} \mathcal{H}^{n-1}(\mathcal{S}(\lambda)) d \lambda \\
\geq & \int_{0}^{\tau^{-2 n} M}\left(t \mathcal{H}^{n-1}(\mathcal{A}(\lambda))^{1 /(n-1)}+(1-t) \mathcal{H}^{n-1}(\mathcal{B}(\lambda))^{1 /(n-1)}\right)^{n-1} d \lambda \\
\geq & \int_{0}^{\tau^{-2 n} M}\left(t \mathcal{H}^{n-1}(\mathcal{A}(\lambda))+(1-t) \mathcal{H}^{n-1}(\mathcal{B}(\lambda))\right) d \lambda \\
& \quad-C \int_{0}^{\tau^{-2 n} M}\left|\mathcal{H}^{n-1}(\mathcal{A}(\lambda))-\mathcal{H}^{n-1}(\mathcal{B}(\lambda))\right|^{2 /(n-1)} d \lambda \\
= & t|A|+(1-t)|B|-C \delta^{\bar{\alpha} /[2(n-1)]} \\
\geq & 1-C \delta^{\bar{\alpha} /[2(n-1)]} .
\end{aligned}
$$

We also observe that, since $K=K^{\natural}$, by Lemma 3.1, (4.11), and [C2, Lemma 4.3], for almost every $\lambda>0$ we have

$$
\begin{aligned}
\left|A \backslash \pi^{-1}(\mathcal{A}(\lambda))\right| & =\left|A^{\natural} \backslash \pi^{-1}\left(\mathcal{A}^{\natural}(\lambda)\right)\right| \\
& \leq\left|K \backslash \pi^{-1}(\mathcal{K}(\lambda))\right|+M \mathcal{H}^{n-1}\left(\mathcal{A}^{\natural}(\lambda) \Delta \mathcal{K}(\lambda)\right) \\
& \leq C \lambda^{2}+M \mathcal{H}^{n-1}\left(\mathcal{A}^{\natural}(\lambda) \Delta \mathcal{K}(\lambda)\right),
\end{aligned}
$$

and analogously for B. Also, by (4.9),

$$
\int_{0}^{\tau^{-2 n} M}\left(\mathcal{H}^{n-1}\left(\mathcal{A}^{\natural}(\lambda) \Delta \mathcal{K}(\lambda)\right)+\mathcal{H}^{n-1}\left(\mathcal{B}^{\natural}(\lambda) \Delta \mathcal{K}(\lambda)\right)\right) d \lambda \leq\left|K \backslash A^{\natural}\right|+\left|K \backslash B^{\natural}\right| \leq C \delta^{\bar{\alpha} / n^{2}}
$$

We set

$$
\eta:=\frac{\bar{\alpha}}{n^{2}}
$$

and we notice that $\eta \leq \min \left\{\frac{\bar{\alpha}}{2(n-1)}, \frac{\bar{\alpha}}{4}\right\}$.

Take $\zeta>0$ to be fixed later. Then by (4.12), (4.14), (4.15), (4.16), and by Chebyshev's inequality, we can find a level

$$
\bar{\lambda} \in\left[\frac{10 \delta^{\zeta}}{\tau}, \frac{20 \delta^{\zeta}}{\tau}\right]
$$

such that

$$
\mathcal{H}^{n-1}(\mathcal{S}(\bar{\lambda})) \leq\left(t \mathcal{H}^{n-1}(\mathcal{A}(\bar{\lambda}))^{1 /(n-1)}+(1-t) \mathcal{H}^{n-1}(\mathcal{B}(\bar{\lambda}))^{1 /(n-1)}\right)^{n-1}+C \delta^{\eta-\zeta},
$$




$$
\begin{gathered}
\left|A \backslash \pi^{-1}(\mathcal{A}(\bar{\lambda}))\right|+\left|B \backslash \pi^{-1}(\mathcal{B}(\bar{\lambda}))\right| \leq C\left(\delta^{2 \zeta}+\delta^{\eta-\zeta}\right), \\
\left|\mathcal{H}^{n-1}(\mathcal{A}(\bar{\lambda}))-\mathcal{H}^{n-1}(\mathcal{B}(\bar{\lambda}))\right| \leq C \delta^{\eta} .
\end{gathered}
$$

In addition, from the properties

$$
\left\{\begin{array}{l}
\left.\mathcal{H}^{n-1}(\mathcal{A}(\lambda)) \leq \tau^{-2 n} M \text { for any } \lambda>0 \text { (see (3.1) }\right) \\
\int_{0}^{\tau^{-2 n} M} \mathcal{H}^{n-1}(\mathcal{A}(\lambda)) d \lambda=|A| \geq 1-\delta \\
\lambda \mapsto \mathcal{H}^{n-1}(\mathcal{A}(\lambda)) \text { is a decreasing function, }
\end{array}\right.
$$

we deduce that

$$
\frac{\tau^{2 n}}{2 M} \leq \mathcal{H}^{n-1}(\mathcal{A}(\lambda)) \leq \frac{M}{\tau^{2 n}} \quad \forall \lambda \in\left(0, \tau^{2 n}(2 M)^{-1}\right) .
$$

The same holds for $B$ and $S$, hence

$$
\mathcal{H}^{n-1}(\mathcal{S}(\bar{\lambda})), \mathcal{H}^{n-1}(\mathcal{A}(\bar{\lambda})), \mathcal{H}^{n-1}(\mathcal{B}(\bar{\lambda})) \in\left[\tau^{2 n}(2 M)^{-1}, \tau^{-2 n} M\right]
$$

provided $\delta \leq \tau^{-N}$ for some large dimensional constant $N$. Set $\rho:=1 / \mathcal{H}^{n-1}(\mathcal{A}(\bar{\lambda}))^{1 /(n-1)} \in$ $[1 / C, C]$, and define

$$
A^{\prime}:=\rho \mathcal{A}(\bar{\lambda}), \quad B^{\prime}:=\rho \mathcal{B}(\bar{\lambda}), \quad S^{\prime}:=\rho \mathcal{S}(\bar{\lambda}) .
$$

By (4.19) and (4.21) we get

$$
\mathcal{H}^{n-1}\left(A^{\prime}\right)=1, \quad\left|\mathcal{H}^{n-1}\left(B^{\prime}\right)-1\right| \leq C \delta^{\eta}, \quad \mathcal{H}^{n-1}\left(S^{\prime}\right) \leq 1+C \delta^{\eta-\zeta} .
$$

while, by (1.2),

$$
\mathcal{H}^{n-1}\left(S^{\prime}\right)^{1 /(n-1)} \geq t \mathcal{H}^{n-1}\left(A^{\prime}\right)^{1 /(n-1)}+(1-t) \mathcal{H}^{n-1}\left(B^{\prime}\right)^{1 /(n-1)} \geq 1-C \delta^{\eta},
$$

therefore

$$
\left|\mathcal{H}^{n-1}\left(A^{\prime}\right)-1\right|+\left|\mathcal{H}^{n-1}\left(B^{\prime}\right)-1\right|+\left|\mathcal{H}^{n-1}\left(S^{\prime}\right)-1\right| \leq C \delta^{\eta-\zeta} .
$$

Thus, by Theorem 1.3 applied with $n-1$, up to a translation there exists a $(n-1)$-dimensional convex set $\Omega^{\prime}$ such that

$$
\Omega^{\prime} \supset A^{\prime} \cup B^{\prime}, \quad \mathcal{H}^{n-1}\left(\Omega^{\prime} \backslash A^{\prime}\right)+\mathcal{H}^{n-1}\left(\Omega^{\prime} \backslash B^{\prime}\right) \leq C \delta^{(\eta-\zeta) \varepsilon_{n-1}(\tau)} .
$$

Define $\zeta$ by

$$
\zeta:=\frac{\varepsilon_{n-1}(\tau)}{3} \eta,
$$

and set $\Omega:=\Omega^{\prime} / \rho$. Then we obtain (recall that $1 / \rho \leq C$ and that $\varepsilon_{n-1}(\tau) \leq 1$ )

$$
\Omega \supset \mathcal{A}(\bar{\lambda}) \cup \mathcal{B}(\bar{\lambda}), \quad \mathcal{H}^{n-1}(\Omega \backslash \mathcal{A}(\bar{\lambda}))+\mathcal{H}^{n-1}(\Omega \backslash \mathcal{B}(\bar{\lambda})) \leq C \delta^{2 \zeta} .
$$


Step 2: Theorem 1.1 applies to most of the sets $A_{y}$ and $B_{y}$ for $y \in \mathcal{A}(\bar{\lambda}) \cap \mathcal{B}(\bar{\lambda})$.

Define $\mathcal{C}:=\mathcal{A}(\bar{\lambda}) \cap \mathcal{B}(\bar{\lambda}) \subset \mathcal{S}(\bar{\lambda})$. By (4.20), (4.23), (3.1), and (4.22), we have

$$
\begin{aligned}
\left|A \backslash \pi^{-1}(\mathcal{C})\right|+\left|B \backslash \pi^{-1}(\mathcal{C})\right| \leq & \left|A \backslash \pi^{-1}(\mathcal{A}(\bar{\lambda}))\right|+\left|B \backslash \pi^{-1}(\mathcal{B}(\bar{\lambda}))\right| \\
& +\int_{(\mathcal{A}(\bar{\lambda})) \backslash(\mathcal{B}(\bar{\lambda}))} \mathcal{H}^{1}\left(A_{y}\right) d y+\int_{(\mathcal{B}(\bar{\lambda})) \backslash(\mathcal{A}(\bar{\lambda}))} \mathcal{H}^{1}\left(B_{y}\right) d y \\
\leq & C\left(\delta^{2 \zeta}+\delta^{\eta-\zeta}\right)+C\left(\mathcal{H}^{n-1}(\Omega \backslash \mathcal{A}(\bar{\lambda}))+\mathcal{H}^{n-1}(\Omega \backslash \mathcal{B}(\bar{\lambda}))\right) \\
\leq & C\left(\delta^{2 \zeta}+\delta^{\eta-\zeta}\right) \leq C \delta^{2 \zeta}
\end{aligned}
$$

Hence, by (1.3) and (4.24),

$$
\begin{aligned}
\int_{\mathcal{C}} \mathcal{H}^{1}\left(S_{y} \backslash\left(t A_{y}+(1-t) B_{y}\right)\right) d y & =\int_{\mathcal{C}}\left[\mathcal{H}^{1}\left(S_{y}\right)-\mathcal{H}^{1}\left(t A_{y}+(1-t) B_{y}\right)\right] d y \\
& \leq \int_{\mathcal{C}}\left[\mathcal{H}^{1}\left(S_{y}\right)-t \mathcal{H}^{1}\left(A_{y}\right)-(1-t) \mathcal{H}^{1}\left(B_{y}\right)\right] d y \\
& =\left|S \cap \pi^{-1}(\mathcal{C})\right|-t\left|A \cap \pi^{-1}(\mathcal{C})\right|-(1-t)\left|B \cap \pi^{-1}(\mathcal{C})\right| \\
& \leq|S|-t|A|-(1-t)|B|+t\left|A \backslash \pi^{-1}(\mathcal{C})\right|+(1-t)\left|B \backslash \pi^{-1}(\mathcal{C})\right| \\
& \leq C \delta^{2 \zeta} .
\end{aligned}
$$

Write $\mathcal{C}$ as $\mathcal{C}_{1} \cup \mathcal{C}_{2}$, where

$$
\mathcal{C}_{1}:=\left\{y \in \mathcal{C}: \mathcal{H}^{1}\left(S_{y}\right)-t \mathcal{H}^{1}\left(A_{y}\right)-(1-t) \mathcal{H}^{1}\left(B_{y}\right) \leq \delta^{\zeta}\right\}, \quad \mathcal{C}_{2}:=\mathcal{C} \backslash \mathcal{C}_{1} .
$$

By Chebyshev's inequality

$$
\mathcal{H}^{n-1}\left(\mathcal{C}_{2}\right) \leq C \delta^{\zeta}
$$

while, recalling (4.18),

$$
\min \left\{\mathcal{H}^{1}\left(A_{y}\right), \mathcal{H}^{1}\left(B_{y}\right)\right\} \geq \bar{\lambda}>\delta^{\zeta} / 2 \quad \forall y \in \mathcal{C}_{1} .
$$

Hence, by Theorem 1.1 applied to $A_{y}, B_{y} \subset \mathbb{R}$ for $y \in \mathcal{C}_{1}$, we deduce that

$$
\mathcal{H}^{1}\left(\operatorname{co}\left(A_{y}\right) \backslash A_{y}\right)+\mathcal{H}^{1}\left(\operatorname{co}\left(B_{y}\right) \backslash B_{y}\right) \leq C \delta^{\zeta}
$$

(recall that $\operatorname{co}(E)$ denotes the convex hull of a set $E$ ). Let $\hat{\mathcal{C}}_{1} \subset \mathcal{C}_{1}$ denote the set of $y \in \mathcal{C}_{1}$ such that

$$
\mathcal{H}^{1}\left(S_{y} \backslash\left(t A_{y}+(1-t) B_{y}\right)\right) \leq \delta^{\zeta},
$$

and notice that, by (4.25) and Chebyshev's inequality, $\mathcal{H}^{n-1}\left(\mathcal{C}_{1} \backslash \hat{\mathcal{C}}_{1}\right) \leq C \delta^{\zeta}$. Then choose a compact set $\overline{\mathcal{C}}_{1} \subset \hat{\mathcal{C}}_{1}$ such that $\mathcal{H}^{n-1}\left(\hat{\mathcal{C}}_{1} \backslash \overline{\mathcal{C}}_{1}\right) \leq \delta^{\zeta}$ to obtain

$$
\mathcal{H}^{n-1}\left(\mathcal{C}_{1} \backslash \overline{\mathcal{C}}_{1}\right) \leq C \delta^{\zeta}
$$

In particular, it follows from (4.23) that

$$
\mathcal{H}^{n-1}\left(\Omega \backslash \overline{\mathcal{C}}_{1}\right) \leq C \delta^{\zeta} .
$$




\section{Step 3: There is $\bar{S} \subset S$ so that $|S \backslash \bar{S}|$ is small and $\bar{S}$ is bounded.}

Define the compact sets

$$
\bar{A}:=\bigcup_{y \in \overline{\mathcal{C}}_{1}} A_{y}, \quad \bar{B}:=\bigcup_{y \in \overline{\mathcal{C}}_{1}} B_{y}, \quad \bar{S}:=\bigcup_{y \in \overline{\mathcal{C}}_{1}} t A_{y}+(1-t) B_{y} .
$$

Note that by (3.1), (4.24), (4.26), (4.29),

$$
|A \Delta \bar{A}|+|B \Delta \bar{B}|=\left|A \backslash \pi^{-1}\left(\overline{\mathcal{C}}_{1}\right)\right|+\left|B \backslash \pi^{-1}\left(\overline{\mathcal{C}}_{1}\right)\right| \leq C \delta^{\zeta},
$$

therefore

$$
|\bar{S}|=\int_{\overline{\mathcal{C}}_{1}} \mathcal{H}^{1}\left(t A_{y}+(1-t) B_{y}\right) d y \geq \int_{\overline{\mathcal{C}}_{1}}\left[t \mathcal{H}^{1}\left(A_{y}\right)+(1-t) \mathcal{H}^{1}\left(B_{y}\right)\right] d y=t|\bar{A}|+(1-t)|\bar{B}| \geq 1-C \delta^{\zeta} .
$$

Hence, by (1.3) (and $\bar{S} \subset S$ ),

$$
|S \Delta \bar{S}| \leq C \delta^{\zeta}
$$

Next we show that $\bar{S}$ is bounded. First recall that

$$
S_{y}=\bigcup_{y=t y^{\prime}+(1-t) y^{\prime \prime}} t A_{y^{\prime}}+(1-t) B_{y^{\prime \prime}}
$$

and by (4.28) we get

$$
\mathcal{H}^{1}\left(\left(\bigcup_{y=t y^{\prime}+(1-t) y^{\prime \prime}} t A_{y^{\prime}}+(1-t) B_{y^{\prime \prime}}\right) \backslash t A_{y}+(1-t) B_{y}\right) \leq \delta^{\zeta} \quad \forall y \in \overline{\mathcal{C}}_{1} .
$$

Recalling that $\bar{\pi}: \mathbb{R}^{n} \rightarrow \mathbb{R}$ is the orthogonal projection onto the last component (that is, $\bar{\pi}(y, s)=$ $s$ ), we define the characteristic functions

$$
\chi_{y}^{A}(s):=\left\{\begin{array}{ll}
1 & \text { if } s \in \bar{\pi}\left(t A_{y}\right) \\
0 & \text { otherwise }
\end{array} \quad \chi_{y}^{A, *}(s):= \begin{cases}1 & \text { if } s \in \bar{\pi}\left(t \operatorname{co}\left(A_{y}\right)\right) \\
0 & \text { otherwise }\end{cases}\right.
$$

and analogously for $B_{y}$ (with $1-t$ in place of $t$ ). Hence, by (4.27) we have the following estimate on the convolution of the functions $\chi_{y}$ and $\chi_{y}^{*}$ :

$$
\begin{aligned}
& \left\|\chi_{y^{\prime}}^{A, *} * \chi_{y^{\prime \prime}}^{B, *}-\chi_{y^{\prime}}^{A} * \chi_{y^{\prime \prime}}^{B}\right\|_{L^{\infty}(\mathbb{R})} \leq\left\|\chi_{y^{\prime \prime}}^{B, *}-\chi_{y^{\prime \prime}}^{B}\right\|_{L^{1}(\mathbb{R})}+\left\|\chi_{y^{\prime}}^{A, *}-\chi_{y^{\prime}}^{A}\right\|_{L^{1}(\mathbb{R})} \\
& \leq \mathcal{H}^{1}\left(\operatorname{co}\left(B_{y^{\prime \prime}}\right) \backslash B_{y^{\prime \prime}}\right)+\mathcal{H}^{1}\left(\operatorname{co}\left(A_{y^{\prime}}\right) \backslash A_{y^{\prime}}\right) \\
& <3 \delta^{\zeta} \quad \forall y^{\prime}, y^{\prime \prime} \in \overline{\mathcal{C}}_{1} \text {. }
\end{aligned}
$$

Let us denote by $[a, b]$ the interval $\bar{\pi}\left(t \operatorname{co}\left(A_{y^{\prime}}\right)+(1-t) \operatorname{co}\left(B_{y^{\prime \prime}}\right)\right)$, and notice that, since by construction

$$
\min \left\{t \mathcal{H}^{1}\left(A_{y}\right),(1-t) \mathcal{H}^{1}\left(B_{y}\right)\right\} \geq \min \{\tau, 1-\tau\} \bar{\lambda} \geq 10 \delta^{\zeta} \quad \forall y \in \overline{\mathcal{C}}_{1}
$$

(see (4.18) ), this interval has length greater than $20 \delta^{\zeta}$. Also, it is easy to check that the function $\chi_{y^{\prime}}^{*} * \chi_{y^{\prime \prime}}^{*}$ is supported on $[a, b]$, has slope equal to 1 (resp. -1 ) inside $\left[a, a+3 \delta^{\zeta}\right]$ (resp. $\left[b-3 \delta^{\zeta}, b\right]$ ), 
and it is greater than $3 \delta^{\zeta}$ inside $\left[a+3 \delta^{\zeta}, b-3 \delta^{\zeta}\right]$. Hence, since $\bar{\pi}\left(t A_{y^{\prime}}+(1-t) B_{y^{\prime \prime}}\right)$ contains the set $\left\{\chi_{y^{\prime}} * \chi_{y^{\prime \prime}}>0\right\}$, by (4.36) we deduce that

$$
\bar{\pi}\left(t A_{y^{\prime}}+(1-t) B_{y^{\prime \prime}}\right) \supset\left[a+3 \delta^{\zeta}, b-3 \delta^{\zeta}\right]
$$

which implies in particular that

$$
\mathcal{H}^{1}\left(t \operatorname{co}\left(A_{y^{\prime}}\right)+(1-t) \operatorname{co}\left(B_{y^{\prime \prime}}\right)\right) \leq \mathcal{H}^{1}\left(t A_{y^{\prime}}+(1-t) B_{y^{\prime \prime}}\right)+6 \delta^{\zeta} \quad \forall y^{\prime}, y^{\prime \prime} \in \overline{\mathcal{C}}_{1} .
$$

We claim that if $y^{\prime}, y^{\prime \prime}, y=t y^{\prime}+(1-t) y^{\prime \prime} \in \overline{\mathcal{C}}_{1}$, then

$$
\bar{\pi}\left(t \operatorname{co}\left(A_{y^{\prime}}\right)+(1-t) \operatorname{co}\left(B_{y^{\prime \prime}}\right)\right) \subset\left[\alpha_{y}-16 \delta^{\zeta}, \beta_{y}+16 \delta^{\zeta}\right],
$$

where $\left[\alpha_{y}, \beta_{y}\right]:=\bar{\pi}\left(t \operatorname{co}\left(A_{y}\right)+(1-t) \operatorname{co}\left(B_{y}\right)\right)$.

Indeed, if this was false, since $\bar{\pi}\left(t \operatorname{co}\left(A_{y^{\prime}}\right)+(1-t) \operatorname{co}\left(B_{y^{\prime \prime}}\right)\right)=[a, b]$ is an interval of length at least $20 \delta^{\zeta} \geq 16 \delta^{\zeta}$, it follows that

$$
\mathcal{H}^{1}\left([a, b] \backslash\left[\alpha_{y}, \beta_{y}\right]\right) \geq 16 \delta^{\zeta}
$$

This implies that

$$
\mathcal{H}^{1}\left(\left[a+3 \delta^{\zeta}, b-3 \delta^{\zeta}\right] \backslash\left[\alpha_{y}, b_{y}\right]\right) \geq 10 \delta^{\zeta},
$$

so, by (4.37),

$$
\mathcal{H}^{1}\left(\bar{\pi}\left(t A_{y^{\prime}}+(1-t) B_{y^{\prime \prime}}\right) \backslash\left[\alpha_{y}, \beta_{y}\right]\right) \geq 10 \delta^{\zeta} .
$$

However, since $\left[\alpha_{y}, \beta_{y}\right] \supset \bar{\pi}\left(t A_{y}+(1-t) B_{y}\right)$, this contradicts (4.35) and proves the claim (4.39).

Now, if we write

$$
\operatorname{co}\left(A_{y}\right)=\{y\} \times\left[a^{A}(y), b^{A}(y)\right], \quad \operatorname{co}\left(B_{y}\right)=\{y\} \times\left[a^{B}(y), b^{B}(y)\right], \quad \operatorname{co}\left(\bar{S}_{y}\right)=\{y\} \times\left[a^{\bar{S}}(y), b^{\bar{S}}(y)\right],
$$

and we denote by $c^{A}(y):=\frac{a^{A}(y)+b^{A}(y)}{2}$ the barycenter of $\operatorname{co}\left(A_{y}\right)$ (and analogously for $B$ and $\bar{S}$ ), then $b^{\bar{S}}=t b^{A}+(1-t) b^{B}$ and it follows from (4.39) that

$$
t b^{A}\left(y^{\prime}\right)+(1-t) b^{B}\left(y^{\prime \prime}\right) \leq b^{\bar{S}}(y)+16 \delta^{\zeta} \quad \forall y, y^{\prime}, y^{\prime \prime} \in \overline{\mathcal{C}}_{1}, y=t y^{\prime}+(1-t) y^{\prime \prime}
$$

(and analogously for $a$ ). Hence, from the fact that $\mathcal{H}^{1}\left(\operatorname{co}\left(A_{y}\right)\right)$ and $\mathcal{H}^{1}\left(\operatorname{co}\left(B_{y}\right)\right)$ are universally bounded (see (3.1) and (4.27) ) one easily deduces that

$$
\left|t c^{A}\left(y^{\prime}\right)+(1-t) c^{B}\left(y^{\prime \prime}\right)-c^{\bar{S}}(y)\right| \leq C \quad \forall y, y^{\prime}, y^{\prime \prime} \in \overline{\mathcal{C}}_{1}, y=t y^{\prime}+(1-t) y^{\prime \prime} .
$$

Hence, by Remark 4.1 in Section 4.1 we get that

$$
\left|c^{A}\left(y_{1}\right)+c^{A}\left(y_{2}\right)-c^{A}\left(t_{A}^{\prime} y_{1}+\left(1-t_{A}^{\prime}\right) y_{2}\right)-c^{A}\left(t_{A}^{\prime \prime} y_{1}+\left(1-t_{A}^{\prime \prime}\right) y_{2}\right)\right| \leq C
$$

whenever $y_{1}, y_{2}, t_{A}^{\prime} y_{1}+\left(1-t_{A}^{\prime}\right) y_{2}, t_{A}^{\prime \prime} y_{1}+\left(1-t_{A}^{\prime \prime}\right) y_{2} \in \overline{\mathcal{C}}_{1}$, with $t_{A}^{\prime}:=\frac{1}{2-t}$ and $t_{A}^{\prime \prime}:=1-t_{A}^{\prime}$. As proved in Lemma 5.1, this estimate in one dimension implies that, along any segment $\left[y_{1}, y_{2}\right]$ 
on which $\overline{\mathcal{C}}_{1}$ has large measure and such that $y_{1}, y_{2}, t_{A}^{\prime} y_{1}+\left(1-t_{A}^{\prime}\right) y_{2}, t_{A}^{\prime \prime} y_{1}+\left(1-t_{A}^{\prime \prime}\right) y_{2} \in \overline{\mathcal{C}}_{1}, c^{A}$ is at bounded distance from a linear function $\ell_{A}$. Analogously,

$$
\left|c^{B}\left(y_{1}\right)+c^{B}\left(y_{2}\right)-c^{B}\left(t_{B}^{\prime} y_{1}+\left(1-t_{B}^{\prime}\right) y_{2}\right)-c^{B}\left(t_{B}^{\prime \prime} y_{1}+\left(1-t_{B}^{\prime \prime}\right) y_{2}\right)\right| \leq C
$$

whenever $y_{1}, y_{2}, t_{B}^{\prime} y_{1}+\left(1-t_{B}^{\prime}\right) y_{2}, t_{B}^{\prime \prime} y_{1}+\left(1-t_{B}^{\prime \prime}\right) y_{2} \in \overline{\mathcal{C}}_{1}$, now with $t_{B}^{\prime}:=\frac{1}{1+t}$ and $t_{B}^{\prime \prime}:=1-t_{B}^{\prime}$ (recall again Remark 4.1), so $c^{B}$ is at bounded distance from a linear function $\ell_{B}$ along any segment $\left[y_{1}, y_{2}\right]$ on which $\overline{\mathcal{C}}_{1}$ has large measure and such that $y_{1}, y_{2}, t_{B}^{\prime} y_{1}+\left(1-t_{B}^{\prime}\right) y_{2}, t_{B}^{\prime \prime} y_{1}+\left(1-t_{B}^{\prime \prime}\right) y_{2} \in \overline{\mathcal{C}}_{1}$. Hence, along any segment $\left[y_{1}, y_{2}\right]$ on which $\overline{\mathcal{C}}_{1}$ has large measure and such that $y_{1}, y_{2}, t_{A}^{\prime} y_{1}+(1-$ $\left.t_{A}^{\prime}\right) y_{2}, t_{A}^{\prime \prime} y_{1}+\left(1-t_{A}^{\prime \prime}\right) y_{2}, t_{B}^{\prime} y_{1}+\left(1-t_{B}^{\prime}\right) y_{2}, t_{B}^{\prime \prime} y_{1}+\left(1-t_{B}^{\prime \prime}\right) y_{2} \in \overline{\mathcal{C}}_{1}, c^{\bar{S}}$ is at bounded distance from the linear function $\ell(y):=t \ell_{A}(y)+(1-t) \ell_{B}(y)$.

We now use this information to deduce that, up to an affine transformation of the form

$$
\mathbb{R}^{n-1} \times \mathbb{R} \ni(y, s) \mapsto(T y, t-L y)+\left(y_{0}, t_{0}\right)
$$

with $T: \mathbb{R}^{n-1} \rightarrow \mathbb{R}^{n-1}$, $\operatorname{det}(T)=1$, and $\left(y_{0}, t_{0}\right) \in \mathbb{R}^{n}$, the set $\bar{S}$ is universally bounded, say $\bar{S} \subset B_{R}$ for some universal constant $R$.

Indeed, first of all, since $\overline{\mathcal{C}}_{1}$ is almost of full measure inside the convex set $\Omega$ (see (4.23), (4.26), and (4.29) ), by a simple Fubini argument (see the analogous argument in [FJ, Proof of Theorem 1.2, Step 3-b] for more details) we can choose $n$ "good" points $y_{1}, \ldots, y_{n} \in \overline{\mathcal{C}}_{1}$ such that:

(a) All points

$$
y_{1}, \ldots, y_{n} \quad \text { and } \quad t_{A}^{\prime} y_{1}+\left(1-t_{A}^{\prime}\right) y_{2}, t_{A}^{\prime \prime} y_{1}+\left(1-t_{A}^{\prime \prime}\right) y_{2}, t_{B}^{\prime} y_{1}+\left(1-t_{B}^{\prime}\right) y_{2}, t_{B}^{\prime \prime} y_{1}+\left(1-t_{B}^{\prime \prime}\right) y_{2}
$$

belong to $\overline{\mathcal{C}_{1}}$.

(b) Let $\Sigma_{i}, i=1, \ldots, n$, denote the $(i-1)$-dimensional simplex generated by $y_{1}, \ldots, y_{i}$, and define

$\Sigma_{i}^{\prime}:=\left[t_{A}^{\prime} \Sigma_{i}+\left(1-t_{A}^{\prime}\right) y_{i+1}\right] \cup\left[t_{A}^{\prime \prime} \Sigma_{i}+\left(1-t_{A}^{\prime \prime}\right) y_{i+1}\right] \cup\left[t_{B}^{\prime} \Sigma_{i}+\left(1-t_{B}^{\prime}\right) y_{i+1}\right] \cup\left[t_{B}^{\prime \prime} \Sigma_{i}+\left(1-t_{B}^{\prime \prime}\right) y_{i+1}\right]$, $i=1, \ldots, n-1$. Then

$$
\mathcal{H}^{i-1}\left(\Sigma_{i}\right) \geq c_{n}, \quad \frac{\mathcal{H}^{i-1}\left(\Sigma_{i} \cap \overline{\mathcal{C}}_{1}\right)}{\mathcal{H}^{i-1}\left(\Sigma_{i}\right)} \geq 1-\delta^{\zeta / 2}, \quad \forall i=2, \ldots, n
$$

$$
\frac{\mathcal{H}^{i-1}\left(\Sigma_{i}^{\prime} \cap \overline{\mathcal{C}}_{1}\right)}{\mathcal{H}^{i-1}\left(\Sigma_{i}^{\prime}\right)} \geq 1-\delta^{\zeta / 2} \quad \forall i=2, \ldots, n-1 .
$$

Then, thanks to John's Lemma [J], up to an affine transformation of the form (4.41) we can assume that

$$
B_{r} \subset \Omega \subset B_{(n-1) r}, \quad 1 / C_{n}<r<C_{n} \text { with } C_{n} \text { dimensional, }
$$

and

$$
\left(y_{k}, 0\right) \in \bar{S}, \quad \forall k=1, \ldots, n
$$


We then prove that $\bar{S}$ is universally bounded as follows: first of all, thanks to (4.42) we only need to show that $\bar{S}$ is bounded in the last variable. Then, by (4.43) and (a) and (b)-(i) above, thanks to Lemma 5.1 we deduce that $c^{\bar{S}}$ is universally bounded on $\Sigma_{2} \cap \overline{\mathcal{C}}_{1}$.

One then iterates this construction: since $c^{\bar{S}}$ is universally bounded on $\Sigma_{2} \cap \overline{\mathcal{C}}_{1}^{\prime}$ and at $y_{3}$, for any point $z \in \Sigma_{2} \cap \overline{\mathcal{C}}_{1}$ such that $t_{A}^{\prime} z+\left(1-t_{A}^{\prime}\right) y_{3}, t_{A}^{\prime \prime} z+\left(1-t_{A}^{\prime \prime}\right) y_{3}, t_{B}^{\prime} z+\left(1-t_{B}^{\prime}\right) y_{3}, t_{B}^{\prime \prime} z+\left(1-t_{B}^{\prime \prime}\right) y_{2} \in \Sigma_{2}^{\prime} \cap \overline{\mathcal{C}}_{1}$ (these are most of the points) we can apply again Lemma 5.1 to deduce that $c^{\bar{S}}$ is universally bounded on the set $\left[z, y_{3}\right] \cap \overline{\mathcal{C}_{1}}$. An iteration of this argument as in [FJ, Proof of Theorem 1.2, Step 3-d] shows that $c^{\bar{S}}$ is universal bounded on a set $\Sigma_{n}^{\prime \prime}$ such that $\mathcal{H}^{n-1}\left(\Sigma_{n} \backslash \Sigma_{n}^{\prime \prime}\right) \leq C \delta^{\zeta / 2}$.

We now conclude as in [FJ, Proof of Theorem 1.2, Step 3-e]: fix a point $\bar{y}_{1} \in \overline{\mathcal{C}}_{1}$. Then we can find another point $\bar{y}_{2} \in \overline{\mathcal{C}}_{1}$ such that $t_{A}^{\prime} \bar{y}_{1}+\left(1-t_{A}^{\prime}\right) \bar{y}_{2}, t_{A}^{\prime \prime} \bar{y}_{1}+\left(1-t_{A}^{\prime \prime}\right) \bar{y}_{2}, t_{B}^{\prime} \bar{y}_{1}+\left(1-t_{B}^{\prime}\right) \bar{y}_{2}, t_{B}^{\prime \prime} \bar{y}_{1}+\left(1-t_{B}^{\prime \prime}\right) \bar{y}_{2} \in$ $\overline{\mathcal{C}}_{1}$, most of the points on the segment $\left[\bar{y}_{1}, \bar{y}_{2}\right]$ belong to $\overline{\mathcal{C}}_{1}$, and $\mathcal{H}^{1}\left(\left[\bar{y}_{1}, \bar{y}_{2}\right] \cap \Sigma_{n}^{\prime \prime}\right) \geq c_{n}^{\prime}$ for some dimensional constant $c_{n}^{\prime}>0$. Hence, on this segment $c^{\bar{S}}$ must be at some bounded distance from a linear function $\ell$, but at the same time we know that $c^{\bar{S}}$ is universally bounded on $\left[\bar{y}_{1}, \bar{y}_{2}\right] \cap \Sigma_{n}^{\prime \prime}$, so $\ell$ is universally bounded there. Since this set has non-trivial measure, this implies that $\ell$ has to be universally bounded on the whole segment $\left[\bar{y}_{1}, \bar{y}_{2}\right]$ (since $\ell$ is a linear function). Thus $c^{\bar{S}}$ is universally bounded on $\left[\bar{y}_{1}, \bar{y}_{2}\right] \cap \overline{\mathcal{C}}_{1}$ as well, and this provides a universal bound for $c^{\bar{S}}\left(\bar{y}_{1}\right)$, concluding the proof.

Step 4: There are uniformly bounded vertically convex sets $A^{\sim}$ and $B^{\sim}$ near $A$ and $B$.

Let $\bar{A}, \bar{B}$, and $\bar{S}$ be as in (4.31), and recall that by the previous step there exists a constant $R$ such that $\bar{S} \subset\left\{\left|x_{n}\right| \leq R\right\}$. Let us apply opposite translations along the $e_{n}$-axis to $t \bar{A}$ and $(1-t) \bar{B}$ (see (4.31)), i.e.,

$$
t \bar{A} \mapsto t \bar{A}+\mu e_{n}, \quad(1-t) \bar{B} \mapsto(1-t) \bar{B}-\mu e_{n},
$$

for some $\mu \in \mathbb{R}$, so that $\bar{A} \subset\left\{x_{n} \geq-R\right\}$ and $\bar{A} \cap\left\{x_{n}=-R\right\} \neq \emptyset$ (recall that $\bar{A}$ is compact). This means that

$$
\min _{y \in \overline{\mathcal{C}}_{1}} a^{A}(y)=-R
$$

Notice that, thanks to (4.39),

$$
t a^{A}\left(y^{\prime}\right)+(1-t) a^{B}\left(y^{\prime \prime}\right) \geq a^{\bar{S}}(y)-16 \delta^{\zeta} \quad \forall y, y^{\prime}, y^{\prime \prime} \in \overline{\mathcal{C}}_{1}, y=t y^{\prime}+(1-t) y^{\prime \prime} .
$$

Let $\bar{y} \in \overline{\mathcal{C}_{1}}$ be such that $a^{A}(\bar{y})=-R$, and set

$$
\mathcal{C}_{1}^{-}:=\overline{\mathcal{C}}_{1} \cap \frac{\overline{\mathcal{C}}_{1}-t \bar{y}}{1-t} .
$$

Then, since $a^{A}(\bar{y})=-R$ and $a^{\bar{S}} \geq-R$, it follows from (4.44) that

$$
a^{B}\left(y^{\prime \prime}\right) \geq-R-C \delta^{\zeta} \geq-R-1 \quad \forall y^{\prime \prime} \in \mathcal{C}_{1}^{-} .
$$

Define

$$
A^{\sim}:=\bigcup_{y \in \mathcal{C}_{1}^{-}}\{y\} \times\left[a^{A}(y), b^{A}(y)\right], \quad B^{\sim}:=\bigcup_{y \in \mathcal{C}_{1}^{-}}\{y\} \times\left[a^{B}(y), b^{B}(y)\right]
$$


We have shown that

$$
A^{\sim} \cup B^{\sim} \subset\left\{x_{n} \geq-R-1\right\}
$$

It remains to prove the upper bounds. Note that because $\bar{y} \in \Omega$ and $\Omega$ is convex, it follows from (4.30) that

$$
\mathcal{H}^{n-1}\left(\Omega \backslash \frac{\overline{\mathcal{C}}_{1}-t \bar{y}}{1-t}\right)=(1-t)^{1-n} \mathcal{H}^{n-1}\left(((1-t) \Omega+t \bar{y}) \backslash \overline{\mathcal{C}}_{1}\right) \leq(1-t)^{1-n} \mathcal{H}^{n-1}\left(\Omega \backslash \overline{\mathcal{C}}_{1}\right) \leq C \delta^{\zeta} .
$$

Therefore, using (4.30) again, we have

$$
\mathcal{H}^{n-1}\left(\Omega \backslash \mathcal{C}_{1}^{-}\right) \leq C \delta^{\zeta} .
$$

We now claim that $A^{\sim} \cup B^{\sim} \subset\left\{x_{n} \leq \tilde{C} R\right\}$ for some universal constant $\tilde{C}$. Indeed, if for instance $b^{A}(\tilde{y}) \geq \tilde{C} R$ for some $\tilde{y} \in \mathcal{C}_{1}^{-}$, then we could use (4.40) and the fact that $b^{B} \geq a^{B} \geq-R-1$ on $\mathcal{C}_{1}^{-}$ to get

$$
b^{\bar{S}}(y) \geq t \tilde{C} R-(1-t)(R+1)-16 \delta^{\zeta} \geq \tau \tilde{C} R-R-2 \quad \forall y \in \overline{\mathcal{C}}_{1} \cap\left(t \tilde{y}+(1-t) \mathcal{C}_{1}^{-}\right),
$$

and since the latter set is nonempty (because of (4.30), (4.45) and the convexity of $\Omega$ ) this contradicts the fact that $b^{\bar{S}} \leq R$ provided $\tilde{C}$ is large enough (the case $b^{B}(\tilde{y}) \geq \tilde{C} R$ for some $\tilde{y} \in \overline{\mathcal{C}}_{1}$ is completely analogous). Thus, $A^{\sim}$ and $B^{\sim}$ are universally bounded.

Finally, note that (3.1), (4.32), (4.45), and (4.27) imply

$$
\left|A \Delta A^{\sim}\right|+\left|B \Delta B^{\sim}\right| \leq C \delta^{\zeta} .
$$

Step 5: The inductive hypothesis applies to horizontal sections and hence there are convex sets close to $A^{\sim}$ and $B^{\sim}$.

The main goal of this section is to show that the hypotheses of Lemma 3.6 apply to the function $b^{A}$ (and similarly to $b^{B},-a^{A}$, and $-a^{B}$ ). The fact that $A^{\sim}$ and $B^{\sim}$ are close to convex sets will then follow easily.

As explained in the outline of the proof in Section 2, to be able to apply Lemma 3.6 we will construct auxiliary sets $A^{-}$and $B^{-}$which consist of the top profile of $A^{\sim}$ and $B^{\sim}$ with a flat bottom, for which the slices coincide with the superlevel sets of $b^{A}$ and $b^{B}$, and we will apply Lemma 3.5 to such sets. However, to be able to do this, we must show that $A^{-}$and $B^{-}$are almost optimal in the Brunn-Minkowski inequality.

As we showed in Step $4, A^{\sim}$ and $B^{\sim}$ are universally bounded, so we may choose universal constants $M_{A} \geq 0$ and $M_{B} \geq 0$ such that

$$
-M_{A} \leq a^{A}(y), \quad-M_{B} \leq a^{B}(y) \quad \forall y \in \mathcal{C}_{1}^{-},
$$

and such that the sets

$$
A^{-}:=\bigcup_{y \in \mathcal{C}_{1}^{-}}\{y\} \times\left[-M_{A}, b^{A}(y)\right], \quad B^{-}:=\bigcup_{y \in \mathcal{C}_{1}^{-}}\{y\} \times\left[-M_{B}, b^{B}(y)\right],
$$


are universally bounded. We may also adjust the constants $M_{A}$ and $M_{B}$ so that $\left|A^{-}\right|=\left|B^{-}\right|$.

Define

$$
S^{-}:=t A^{-}+(1-t) B^{-} ; \quad \mathcal{C}^{-}(y):=\left\{\left(y^{\prime}, y^{\prime \prime}\right) \in \mathcal{C}^{-} \times \mathcal{C}^{-}: t y^{\prime}+(1-t) y^{\prime \prime}=y\right\} .
$$

We estimate the measure of $S^{-}$using (4.40) as follows:

$$
\begin{aligned}
\left|S^{-}\right| & =\int_{t \mathcal{C}_{1}^{-}+(1-t) \mathcal{C}_{1}^{-}} \mathcal{H}^{1}\left(\bigcup_{\left(y^{\prime}, y^{\prime \prime}\right) \in \mathcal{C}^{-}(y)} t\left[-M_{A}, b^{A}\left(y^{\prime}\right)\right]+(1-t)\left[-M_{B}, b^{B}\left(y^{\prime \prime}\right)\right]\right) d y \\
& \leq \int_{t \mathcal{C}_{1}^{-}+(1-t) \mathcal{C}_{1}^{-}}\left(b^{\bar{S}}(y)+16 \delta^{\zeta}+t M_{A}+(1-t) M_{B}\right) d y \\
& \leq \int_{\mathcal{C}_{1}^{-}}\left(b^{\bar{S}}(y)+t M_{A}+(1-t) M_{B}\right) d y+C \delta^{\zeta},
\end{aligned}
$$

where, in the final inequality, we used that $\mathcal{H}^{n-1}\left(\left(t \mathcal{C}_{1}^{-}+(1-t) \mathcal{C}_{1}^{-}\right) \backslash \mathcal{C}_{1}^{-}\right) \leq C \delta^{\zeta}$ (recall that $\mathcal{C}_{1}^{-} \subset \Omega$ and $\Omega$ is convex, thus $t \mathcal{C}_{1}^{-}+(1-t) \mathcal{C}_{1}^{-} \subset \Omega$ and the bound follows from (4.45)) and that $b^{\bar{S}}$ is universally bounded on $\mathcal{C}_{1}^{-}$. Next, since $b^{\bar{S}}=t b^{A}+(1-t) b^{B}$ and $\left|A^{-}\right|=\left|B^{-}\right|$, it follows that

$$
\begin{aligned}
\left|S^{-}\right| & \leq t \int_{\mathcal{C}_{1}^{-}}\left(b^{A}(y)-M_{A}\right) d y+(1-t) \int_{\mathcal{C}_{1}^{-}}\left(b^{B}(y)-M_{B}\right) d y+C \delta^{\zeta} \\
& =t\left|A^{-}\right|+(1-t)\left|B^{-}\right|+C \delta^{\zeta}=\left|A^{-}\right|+C \delta^{\zeta},
\end{aligned}
$$

On the other hand (1.2) implies

$$
\left|S^{-}\right| \geq\left(t\left|A^{-}\right|^{1 / n}+(1-t)\left|B^{-}\right|^{1 / n}\right)^{n}=\left|A^{-}\right| .
$$

Hence, in all, we find that

$$
0 \leq\left|S^{-}\right|-\left|A^{-}\right| \leq C \delta^{\zeta} \text { and }\left|A^{-}\right|=\left|B^{-}\right|
$$

We are now in a position to apply Lemma 3.5 to $A^{-}$and $B^{-}$to confirm that hypothesis (3.10) of Lemma 3.6 is valid for $b^{A}$ and $b^{B}$.

Let us recall the notation $E(s) \subset \mathbb{R}^{n-1} \times\{s\}$ in (2.1). Since $\left|c A^{-}\right|=\left|c B^{-}\right|=1$ for some universal constant $c>0$, by applying (4.47) and Lemma 3.5 to the sets $c A^{-}, c B^{-}$, and $c S^{-}$, we find a monotone map $T: \mathbb{R} \rightarrow \mathbb{R}$ such that

$$
\begin{gathered}
T_{\sharp} \rho_{A^{-}}=\rho_{B^{-}}, \quad \rho_{A^{-}}(s):=\frac{\mathcal{H}^{n-1}\left(A^{-}(s)\right)}{\left|A^{-}\right|}, \quad \rho_{B^{-}}(s):=\frac{\mathcal{H}^{n-1}\left(B^{-}(s)\right)}{\left|B^{-}\right|}, \\
T^{\prime}(s)=\frac{\mathcal{H}^{n-1}\left(A^{-}(s)\right)\left|B^{-}\right|}{\mathcal{H}^{n-1}\left(B^{-}(T(s))\right) \mid A^{-1}} \quad \rho_{A^{-} \text {-a.e. },} \\
\int_{\mathbb{R}} e_{n-1}(s)\left[t+(1-t) T^{\prime}(s)\right] d s \leq C \delta^{\zeta},
\end{gathered}
$$


and

$$
\int_{\mathbb{R}}\left|\frac{\rho_{A^{-}}(s)}{\rho_{B^{-}}(T(s))}-1\right| \rho_{A^{-}}(s) d s \leq C \delta^{\zeta / 2}
$$

where $T_{t}(s)=t s+(1-t) T(s)$ and

$$
e_{n-1}(s):=\mathcal{H}^{n-1}\left(S^{-}\left(T_{t}(s)\right)\right)-\left[t \mathcal{H}^{n-1}\left(A^{-}(s)\right)^{1 /(n-1)}+(1-t) \mathcal{H}^{n-1}\left(B^{-}(T(s))\right)^{1 /(n-1)}\right]^{n-1} .
$$

Let us define the set

$$
G:=\left\{s \in \mathbb{R}: e_{n-1}(s) \leq \delta^{\zeta / 2}\right\}
$$

and observe that, thanks to (4.49), (4.51), and $T^{\prime} \geq 0$,

$$
\mathcal{H}^{1}(\mathbb{R} \backslash G) \leq \frac{1}{\tau} \int_{\mathbb{R} \backslash G}\left[t+(1-t) T^{\prime}(s)\right] d s \leq C \delta^{\zeta / 2} .
$$

Next, note that the formula for $T$ (with $A$ and $B$ replaced by $A^{-}$and $B^{-}$) given in the footnote in the statement of Lemma 3.5 implies that the distributional derivative of $T$ has no singular part on $T^{-1}\left(\left\{\rho_{B^{-}}>0\right\}\right)$. Hence, the area formula gives

$$
\mathcal{H}^{1}\left((\mathbb{R} \backslash T(G)) \cap\left\{\rho_{B}>0\right\}\right)=\int_{(\mathbb{R} \backslash G) \cap T^{-1}\left(\left\{\rho_{B}>0\right\}\right)} T^{\prime}(s) d s,
$$

and it follows that

$$
\mathcal{H}^{1}\left((\mathbb{R} \backslash T(G)) \cap\left\{\rho_{B^{-}}>0\right\}\right) \leq \int_{\mathbb{R} \backslash G} T^{\prime}(s) d s \leq \frac{1}{\tau} \int_{\mathbb{R} \backslash G}\left[t+(1-t) T^{\prime}(s)\right] d s \leq C \delta^{\zeta / 2} .
$$

Also, we define

$$
I_{A^{-}}:=\left\{s \in \mathbb{R}: \mathcal{H}^{n-1}\left(A^{-}(s)\right)>\delta^{\zeta / 4}\right\}, \quad I_{B^{-}}:=\left\{s \in \mathbb{R}:, \mathcal{H}^{n-1}\left(B^{-}(s)\right)>\delta^{\zeta / 4}\right\}
$$

and

$$
I_{T}:=\left\{s \in \mathbb{R}: \frac{2}{3} \leq \frac{\rho_{A^{-}}(s)}{\rho_{B^{-}}(T(s))} \leq \frac{3}{2}\right\} .
$$

Notice that, thanks to (4.50),

$$
\int_{I_{T}} \rho_{A^{-}}(s) d s \geq 1-C \delta^{\zeta / 2} .
$$

Also, since $\rho_{A^{-}}$and $\rho_{B^{-}}$are probability densities supported inside some bounded interval (being $A^{-}$and $B^{-}$universally bounded), we have

$$
\int_{I_{A^{-}}} \rho_{A^{-}}(s) d s=\int_{\left\{\rho_{A^{-}}>\delta^{\zeta / 4}\left|A^{-}\right|\right\}} \rho_{A^{-}}(s) d s \geq 1-C \delta^{\zeta / 4}
$$

and (using the condition $T_{\sharp} \rho_{A^{-}}=\rho_{B^{-}}$)

$$
\int_{T^{-1}\left(I_{B^{-}}\right)} \rho_{A^{-}}(s) d s=\int_{I_{B^{-}}} \rho_{B^{-}}(s) d s=\int_{22} \rho_{\left\{\rho_{B^{-}}>\delta^{\zeta / 4}\left|B^{-}\right|\right\}} \rho_{B^{-}}(s) d s \geq 1-C \delta^{\zeta / 4} .
$$


Therefore

$$
\int_{I} \rho_{A^{-}}(s) d s=\int_{T(I)} \rho_{B^{-}}(s) d s \geq 1-C \delta^{\zeta / 4}, \quad I:=I_{A^{-}} \cap T^{-1}\left(I_{B^{-}}\right) \cap I_{T} .
$$

We now apply the inductive hypothesis to $A^{-}(s), B^{-}(T(s)), S^{-}\left(T_{t}(s)\right)$ : define

$$
\begin{aligned}
A^{s} & :=\frac{A^{-}(s)}{H^{n-1}\left(A^{-}(s)\right)^{1 /(n-1)}}, \quad B^{s}:=\frac{B^{-}(T(s))}{H^{n-1}\left(B^{-}(T(s))\right)^{1 /(n-1)}}, \\
S^{s} & :=\frac{S^{-}\left(T_{t}(s)\right)}{t H^{n-1}\left(A^{-}(s)\right)^{1 /(n-1)}+(1-t) H^{n-1}\left(B^{-}(T(s))\right)^{1 /(n-1)}}, \\
t^{s} & :=\frac{t H^{n-1}\left(A^{-}(s)\right)^{1 /(n-1)}}{t H^{n-1}\left(A^{-}(s)\right)^{1 /(n-1)}+(1-t) H^{n-1}\left(B^{-}(T(s))\right)^{1 /(n-1)}} .
\end{aligned}
$$

Then, since $\frac{2}{3} \leq \frac{\rho_{A^{-}}(s)}{\rho_{B^{-}}(T(s))} \leq \frac{3}{2}$ for $s \in I$, and $\left|A^{-}\right|=\left|B^{-}\right|$, it follows that

$$
t^{s} \in\left[\frac{\tau}{2}, 1-\frac{\tau}{2}\right] \quad \forall s \in I .
$$

In addition, recalling the definition of $G$, for any $s \in I \cap G$ we also have

$$
S^{s}=t^{s} A^{s}+\left(1-t^{s}\right) B^{s}, \quad \mathcal{H}^{n-1}\left(A^{s}\right)=\mathcal{H}^{n-1}\left(B^{s}\right)=1, \quad \mathcal{H}^{n-1}\left(S^{s}\right) \leq 1+\delta^{\zeta / 4} .
$$

Hence, if

$$
\delta^{\zeta / 4} \leq e^{-M_{n-1}(\tau / 2)},
$$

then by the inductive hypothesis we deduce the existence of a convex set $K^{s}$ such that, up to a translation (which may depend on $s$ )

$$
K^{s} \supset A^{s} \cup B^{s}, \quad \mathcal{H}^{n-1}\left(K^{s} \backslash A^{s}\right)+\mathcal{H}^{n-1}\left(K^{s} \backslash B^{s}\right) \leq C \delta^{\frac{\zeta}{4} \varepsilon_{n-1}(\tau / 2)} .
$$

Thus, in particular,

$$
\mathcal{H}^{n-1}\left(\operatorname{co}\left(A^{s}\right) \backslash A^{s}\right)+\mathcal{H}^{n-1}\left(\operatorname{co}\left(B^{s}\right) \backslash B^{s}\right) \leq \delta^{\frac{\zeta}{4}} \varepsilon_{n-1}(\tau / 2),
$$

which implies that

$$
\mathcal{H}^{n-1}\left(\operatorname{co}\left(A^{-}(s)\right) \backslash A^{-}(s)\right)+\mathcal{H}^{n-1}\left(\operatorname{co}\left(B^{-}(T(s))\right) \backslash B^{-}(T(s))\right) \leq \delta^{\frac{\zeta}{4}} \varepsilon_{n-1}(\tau / 2) \quad \forall s \in I \cap G .
$$

Hence, integrating with respect to $s \in I \cap G$ and using that $T^{\prime} \leq C$ on $I \cap G$ (as a consequence of (4.48) and the fact that $I \subset I_{T}$ ) we obtain

$$
\int_{I \cap G} \mathcal{H}^{n-1}\left(\operatorname{co}\left(A^{-}(s)\right) \backslash A^{-}(s)\right) d s \leq C \delta^{\frac{\zeta}{4} \varepsilon_{n-1}(\tau / 2)},
$$




$$
\begin{aligned}
\int_{T(I \cap G)} \mathcal{H}^{n-1}\left(\operatorname{co}\left(B^{-}(s)\right) \backslash B^{-}(s)\right) d s & =\int_{I \cap G} \mathcal{H}^{n-1}\left(\operatorname{co}\left(B^{-}(T(s))\right) \backslash B^{-}(T(s))\right) T^{\prime}(s) d s \\
& \leq C \delta^{\frac{\zeta}{4} \varepsilon_{n-1}(\tau / 2)} .
\end{aligned}
$$

Also, recalling the definition of $\rho_{A^{-}}$and $\rho_{B^{-}}$, it follows from (4.54), (4.52), (4.53), that

$$
\int_{\mathbb{R} \backslash(I \cap G)} \mathcal{H}^{n-1}\left(A^{-}(s)\right) d s+\int_{\mathbb{R} \backslash(T(I \cap G))} \mathcal{H}^{n-1}\left(B^{-}(s)\right) d s \leq C \delta^{\zeta / 4}
$$

(notice that $B^{-}(s)=\emptyset$ on $\left.\left\{\rho_{B}=0\right\}\right)$.

By the bound above, (4.40), (4.44), (4.54), (4.56), (4.57), and Remark 4.1 (see Section 4.1), we can apply Lemma 3.6 to $b^{A}$ find a concave function $\Psi^{+}(y)$ defined on $\Omega$ such that

$$
\int_{\mathcal{C}_{1}^{-}}\left|b^{A}(y)-\Psi^{+}(y)\right| d y \leq C \delta^{\frac{\zeta \beta_{n, \tau}}{4} \varepsilon_{n-1}(\tau / 2)} .
$$

Similarly, there is a convex function $\Psi^{-}$on $\Omega$ such that

$$
\int_{\mathcal{C}_{1}^{-}}\left|a^{A}(y)-\Psi^{-}(y)\right| d y \leq C \delta^{\frac{\zeta \beta_{n, \tau}}{4} \varepsilon_{n-1}(\tau / 2)},
$$

so the convex set

$$
K_{A}:=\left\{(y, s): y \in \Omega, \Psi^{-}(y) \leq s \leq \Psi^{+}(y)\right\}
$$

satisfies $\left|A^{\sim} \Delta K_{A}\right| \leq C \delta^{\frac{\zeta \beta_{n, \tau}}{4} \varepsilon_{n-1}(\tau / 2)}$. The same argument also applies to $B^{-}$so that, in all, we have

$$
\left|A^{\sim} \Delta K_{A}\right|+\left|B^{\sim} \Delta K_{B}\right| \leq C \delta^{\frac{\zeta \beta_{n, \tau}}{4} \varepsilon_{n-1}(\tau / 2)} .
$$

\section{Step 6: Conclusion.}

By (4.58) and (4.46), we can apply Proposition 3.4 to deduce that, up to a translation, there exists a convex set $\mathcal{K}$ convex such that $A \cup B \subset \mathcal{K}$ and

$$
|\mathcal{K} \backslash A|+|\mathcal{K} \backslash B| \leq C \delta^{\frac{\zeta \beta_{n, \tau}}{8 n^{3}} \varepsilon_{n-1}(\tau / 2)},
$$

concluding the proof.

\section{Step 7: An explicit bound for $\varepsilon_{n}(\tau)$ and $M_{n}(\tau)$.}

By (4.59) and (4.55) it follows that the recurrence for $\varepsilon_{n}(\tau)$ and $M_{n}(\tau)$ is given, respectively, by

$$
\varepsilon_{n}(\tau)=\frac{\zeta \beta_{n, \tau}}{8 n^{2}} \varepsilon_{n-1}(\tau / 2), \quad M_{n}(\tau)=\frac{4}{\zeta} M_{n-1}(\tau / 2) .
$$

Recall that (see (4.22) and Lemma 3.6)

$$
\zeta=\frac{\varepsilon_{n-1}(\tau)}{3} \eta, \quad \beta_{n, \tau}=\frac{\tau}{16(n-1)|\log (\tau)|} .
$$


For $n=1$, Theorem 1.1 implies that if $\delta<\tau / 2$, then

$$
|\mathcal{K} \backslash A|+|\mathcal{K} \backslash B| \leq 8 \delta / \tau .
$$

In other words, $\varepsilon_{1}(\tau)=1$ and $M_{1}(\tau)=|\log (\tau / 3)|$ are admissible choices.

For $n \geq 2$ we have (see (4.10) and (4.17))

$$
\eta=\frac{\bar{\alpha}}{n^{2}}=\frac{\beta_{n, \tau}}{2^{4} n^{2}}=\frac{\tau}{2^{7}(n-1) n^{2}|\log \tau|},
$$

thus

$$
\zeta=\frac{\tau}{2^{7} \cdot 3(n-1) n^{2}|\log \tau|} \varepsilon_{n-1}(\tau)
$$

which gives

$$
\varepsilon_{n}(\tau)=\frac{\tau^{2}}{2^{14} \cdot 3(n-1)^{2} n^{4}|\log \tau|^{2}} \varepsilon_{n-1}(\tau) \varepsilon_{n-1}(\tau / 2) .
$$

In particular, we obtain and explicit lower bound for all $n \geq 2$ (which can be easily checked to hold by induction):

$$
\varepsilon_{n}(\tau) \geq \frac{\tau^{3^{n}}}{2^{3^{n+1}} n^{3^{n}}|\log \tau|^{3^{n}}} .
$$

Concerning $M_{n}(\tau)$ we have

$$
M_{n}(\tau)=\frac{4}{\zeta} M_{n-1}(\tau / 2)=\frac{2^{9} \cdot 3(n-1) n^{2}|\log \tau|}{\tau \varepsilon_{n-1}(\tau)} M_{n-1}(\tau / 2)
$$

from which we get

$$
M_{n}(\tau) \leq \frac{2^{3^{n+2}} n^{3^{n}}|\log \tau|^{3^{n}}}{\tau^{3^{n}}}
$$

\section{Proof of the technical results}

As in the previous section, we use $C$ to denote a generic constant, which may change from line to line, and that is bounded from above by $\tau^{-N_{n}}$ for some dimensional constant $N_{n}>1$. Again, we will say that such a constant is universal.

\subsection{Proof of Lemma 3.5: an inductive proof of the Brunn-Minkowski inequality}

In this section we show how to prove the Brunn-Minkowski inequality (1.2) by induction on dimension 3 As a byproduct of our proof we obtain the bounds (3.4) and (3.5).

Given compact sets $A, B \subset \mathbb{R}^{n}$ and $S:=t A+(1-t) B$, we define the probability densities on the real line $\rho_{A}, \rho_{B}$, and $\rho_{S}$ as in (3.3), and we let $T: \mathbb{R} \rightarrow \mathbb{R}$ be the monotone rearrangement sending

\footnotetext{
${ }^{3}$ The one-dimensional case is elementary, and can be proved for instance as follows: given $A, B \subset \mathbb{R}$ compact, after translation we can assume that

$$
A \subset(-\infty, 0], \quad B \subset[0,+\infty), \quad A \cap B=\{0\} .
$$

Then $A+B \supset A \cup B$, hence $|A+B| \geq|A \cup B|=|A|+|B|$, as desired.
} 
$\rho_{A}$ onto $\rho_{B}$. Since monotone functions are differentiable almost everywhere, as a consequence of the Area Formula one has (see for instance [AGS, Lemma 5.5.3])

$$
T^{\prime}(s)=\frac{\rho_{A}(s)}{\rho_{B}(T(s))} \quad \rho_{A} \text {-a.e. }
$$

Set $T_{t}(s):=t s+(1-t) T(s)$ and observe that $S\left(T_{t}(s)\right) \supset t A(s)+(1-t) B(T(s))$, so by the Brunn-Minkowski inequality in $\mathbb{R}^{n-1}$ we get

$$
\mathcal{H}^{n-1}\left(S\left(T_{t}(s)\right)\right)^{1 /(n-1)} \geq t \mathcal{H}^{n-1}(A(s))^{1 /(n-1)}+(1-t) \mathcal{H}^{n-1}(B(T(s)))^{1 /(n-1)} .
$$

We now write

$$
\begin{aligned}
|S| & =|S| \int_{\mathbb{R}} \rho_{S}(s) d s \geq|S| \int_{\mathbb{R}} \rho_{S}\left(T_{t}(s)\right) T_{t}^{\prime}(s) d s \\
& =\int_{\mathbb{R}} \mathcal{H}^{n-1}\left(S\left(T_{t}(s)\right)\right)\left[t+(1-t) T^{\prime}(s)\right] d s,
\end{aligned}
$$

(Here, when we applied the change of variable $s \mapsto T_{t}(s)$, we used the fact that since $T_{t}$ is increasing, its pointwise derivative is bounded from above by its distributional derivative.)

Define

$$
\mu_{1}(s)=\ldots=\mu_{n-1}(s):=\frac{1-t}{t} \frac{|B|^{1 /(n-1)} \rho_{B}(T(s))^{1 /(n-1)}}{|A|^{1 /(n-1)} \rho_{A}(s)^{1 /(n-1)}}, \quad \mu_{n}(s):=\frac{1-t}{t} \frac{\rho_{A}(s)}{\rho_{B}(T(s))}
$$

Using (5.2) and (5.1), we obtain

$$
\begin{aligned}
|S| & \geq \int_{\mathbb{R}}\left(t \mathcal{H}^{n-1}(A(s))^{1 /(n-1)}+(1-t) \mathcal{H}^{n-1}(B(T(s)))^{1 /(n-1)}\right)^{n-1}\left[t+(1-t) T^{\prime}(s)\right] d s \\
& =\int_{\mathbb{R}}\left(t|A|^{1 /(n-1)} \rho_{A}(s)^{1 /(n-1)}+(1-t)|B|^{1 /(n-1)} \rho_{B}(T(s))^{1 /(n-1)}\right)^{n-1}\left(t+(1-t) \frac{\rho_{A}(s)}{\rho_{B}(T(s))}\right) d s \\
& =|A| \int_{\mathbb{R}} t^{n} \prod_{i=1}^{n}\left(1+\mu_{i}(s)\right) \rho_{A}(s) d s .
\end{aligned}
$$

We now use the following inequality, see [FMP2, Equation (22)] and [FMP1, Lemma 2.5]: there exists a dimensional constant $c(n)>0$ such that, for any choice of nonnegative numbers $\left\{\mu_{i}\right\}_{i=1, \ldots, n}$,

$$
\prod_{i=1}^{n}\left(1+\mu_{i}\right) \geq\left(1+\left(\prod_{i=1}^{n} \mu_{i}\right)^{1 / n}\right)^{n}+c(n) \frac{1}{\max _{i} \mu_{i}} \sum_{j=1}^{n}\left(\mu_{j}-\left(\prod_{i=1}^{n} \mu_{i}\right)^{1 / n}\right)^{2} .
$$

Hence, we get

$$
\prod_{i=1}^{n}\left(1+\mu_{i}(s)\right) \geq\left(1+\frac{1-t}{t} \frac{|B|^{1 / n}}{|A|^{1 / n}}\right)^{n}+c(n) \frac{1}{\max _{i} \mu_{i}(s)} \sum_{j=1}^{n}\left(\mu_{j}(s)-\frac{1-t}{t} \frac{|B|^{1 / n}}{|A|^{1 / n}}\right)^{2},
$$


which gives (recall that $\int \rho_{A}=1$ )

$$
\begin{aligned}
|S| \geq \mid & |A| \int_{\mathbb{R}} t^{n}\left(1+\frac{1-t}{t} \frac{|B|^{1 / n}}{|A|^{1 / n}}\right)^{n} \rho_{A}(s) d s \\
& \quad+c(n)|A| t^{n} \int_{\mathbb{R}} \frac{1}{\max _{i} \mu_{i}(s)} \sum_{j=1}^{n}\left(\mu_{j}(s)-\frac{1-t}{t} \frac{|B|^{1 / n}}{|A|^{1 / n}}\right)^{2} \rho_{A}(s) d s \\
\geq & \left(t|A|^{1 / n}+(1-t)|B|^{1 / n}\right)^{n},
\end{aligned}
$$

which proves the validity of Brunn-Minkowski in dimension $n$. As a byproduct of this proof we will deduce (3.4) and (3.5).

Indeed (3.4) is immediate from our proof. Moreover, we have

$$
|S|-\left(t|A|^{1 / n}+(1-t)|B|^{1 / n}\right)^{n} \geq c(n)|A| t^{n} \int_{\mathbb{R}} \frac{1}{\max _{i} \mu_{i}(s)} \sum_{j=1}^{n}\left(\mu_{j}(s)-\frac{1-t}{t} \frac{|B|^{1 / n}}{|A|^{1 / n}}\right)^{2} \rho_{A}(s) d s .
$$

With the further assumption (1.3), (5.4) gives

$$
\begin{aligned}
& \int_{\mathbb{R}} \frac{1}{\max _{i} \mu_{i}(s)}\left(\sum_{j=1}^{n}\left|\frac{t}{1-t} \mu_{j}(s)-\frac{|B|^{1 / n}}{|A|^{1 / n}}\right|\right)^{2} \rho_{A}(s) d s \\
& \leq n \int_{\mathbb{R}} \frac{1}{\max _{i} \mu_{i}(s)} \sum_{j=1}^{n}\left(\frac{t}{1-t} \mu_{j}(s)-\frac{|B|^{1 / n}}{|A|^{1 / n}}\right)^{2} \rho_{A}(s) d s \\
& \leq \frac{C(n)}{t^{n-2}(1-t)^{2}} \delta \leq \frac{C(n)}{\tau^{n}} \delta,
\end{aligned}
$$

which, combined with the Schwarz inequality, leads to

$$
\begin{aligned}
\int_{\mathbb{R}} \sum_{j=1}^{n} \mid & \frac{t}{1-t} \mu_{j}(s)-\frac{|B|^{1 / n}}{|A|^{1 / n} \mid} \rho_{A}(s) d s \\
& \leq \frac{C(n)}{\tau^{n / 2}} \delta^{1 / 2} \sqrt{\int_{\mathbb{R}} \max _{i} \mu_{i}(s) \rho_{A}(s) d s} \\
& \leq \frac{C(n)}{\tau^{n / 2}} \delta^{1 / 2}\left(\sqrt{\int_{\mathbb{R}} \max _{i}\left|\frac{t}{1-t} \mu_{i}(s)-\frac{|B|^{1 / n}}{|A|^{1 / n}}\right| \rho_{A}(s) d s}+\sqrt{\frac{|B|^{1 / n}}{|A|^{1 / n}}}\right) \\
& \leq \frac{C(n)}{\tau^{n / 2}} \delta^{1 / 2}\left(\sqrt{\int_{\mathbb{R}} \sum_{j=1}^{n}\left|\frac{t}{1-t} \mu_{j}(s)-\frac{|B|^{1 / n}}{|A|^{1 / n}}\right| \rho_{A}(s) d s}+2\right) .
\end{aligned}
$$

Hence, provided $\delta / \tau^{n}$ is sufficiently small we get

$$
\int_{\mathbb{R}} \sum_{j=1}^{n}\left|\frac{t}{1-t} \mu_{j}(s)-\frac{|B|^{1 / n}}{|A|^{1 / n}}\right| d s \leq \frac{C(n)}{\tau^{n / 2}} \delta^{1 / 2} .
$$

Recalling the definition of $\mu_{i}$ (see (5.3)) and using that $1-4 \delta \leq|B| /|A| \leq 1+4 \delta$, we deduce that (3.5) holds. 


\subsection{Proof of Lemma 3.6}

We first remark that it suffices to prove the result in the case $\hat{M}=1$, since the general case follows by applying the result to the function $f / \hat{M}$. The proof of this result is rather involved and is divided into several steps.

\section{Step a: Making $\psi$ uniformly concave at points that are well separated}

Let $\beta \in(0,1 / 3]$ to be fixed later, and define $\varphi: \Omega \rightarrow \mathbb{R}$ as

$$
\varphi(y):= \begin{cases}\psi(y)+2-20(\sigma+\varsigma)^{\beta}|y|^{2} & y \in F \\ 0 & y \in \Omega \backslash F .\end{cases}
$$

Notice that,

$$
\left|y_{12}^{\prime}\right|^{2}+\left|y_{12}^{\prime \prime}\right|^{2}-\left|y_{1}\right|^{2}-\left|y_{2}\right|^{2}=-2 t^{\prime}\left(1-t^{\prime}\right)\left|y_{1}-y_{2}\right|^{2} \leq-\frac{\tau}{2}\left|y_{1}-y_{2}\right|^{2} .
$$

Because of this, (3.9) and (3.6), we have $0 \leq \varphi \leq 3$ and

$$
\varphi\left(y_{1}\right)+\varphi\left(y_{2}\right) \leq \varphi\left(y_{12}^{\prime}\right)+\varphi\left(y_{12}^{\prime \prime}\right)+\sigma-10 \tau(\sigma+\varsigma)^{\beta}\left|y_{1}-y_{2}\right|^{2} \quad \forall y_{1}, y_{2}, y_{12}^{\prime}, y_{12}^{\prime \prime} \in F,
$$

which implies in particular that

$$
\varphi\left(y_{1}\right)+\varphi\left(y_{2}\right) \leq \varphi\left(y_{12}^{\prime}\right)+\varphi\left(y_{12}^{\prime \prime}\right)+\sigma \quad \forall y_{1}, y_{2}, y_{12}^{\prime}, y_{12}^{\prime \prime} \in F .
$$

Also, since $\beta \leq 1 / 3$,

$\varphi\left(y_{1}\right)+\varphi\left(y_{2}\right)<\varphi\left(y_{12}^{\prime}\right)+\varphi\left(y_{12}^{\prime \prime}\right)-\tau(\sigma+\varsigma)^{\beta}\left|y_{1}-y_{2}\right|^{2} \quad \forall y_{1}, y_{2}, y_{12}^{\prime}, y_{12}^{\prime \prime} \in F,\left|y_{1}-y_{2}\right| \geq \frac{(\sigma+\varsigma)^{\beta}}{\sqrt{\tau}}$

that is $\varphi$ is uniformly concave on points of $F$ that are at least $(\sigma+\varsigma)^{\beta} / \sqrt{\tau}$-apart.

\section{Step b: Constructing a concave function that should be close to $\varphi$}

Let us take $\gamma \in(0, \beta]$ to be fixed later, and define

$$
\bar{\varphi}(y):=\min \{\varphi(y), h\},
$$

where $h \in[0,3]$ is given by

$$
h:=\inf \left\{t>0: \mathcal{H}^{n-1}(\{\varphi>t\}) \leq(\sigma+\varsigma)^{\gamma}\right\} .
$$

Since $0 \leq \varphi \leq 3$, we get

$$
\int_{\Omega}[\varphi(y)-\bar{\varphi}(y)] d y=\int_{h}^{3 M} \mathcal{H}^{n-1}(\{\varphi>s\}) d s \leq 3 M(\sigma+\varsigma)^{\gamma} .
$$

Notice that whenever $\max \left\{\varphi\left(y_{12}^{\prime}\right), \varphi\left(y_{12}^{\prime \prime}\right)\right\} \leq h, \bar{\varphi}$ satisfies (5.6) and (5.7).

We define $\Phi: \Omega \rightarrow[0, h]$ to be the concave envelope of $\bar{\varphi}$, that is, the infimum among all linear functions that are above $\bar{\varphi}$ in $\Omega$. Our goal is to show that $\Phi$ is $L^{1}$-close to $\bar{\varphi}$ (and hence to $\varphi$ ). 
Step c: The set $\{\Phi=\bar{\varphi}\}$ is $K(\sigma+\varsigma)^{\beta}$ dense in $\Omega \backslash \operatorname{co}\left(\left\{\bar{\varphi}>h-K(\sigma+\varsigma)^{\beta}\right\}\right)$.

Let $\beta \in(0,1 / 3]$ be as in Step b. We claim that there exists a universal constant $K>0$ such that the following holds, provided $\beta$ is sufficiently small (chosen later depending on $\tau$ and dimension): For any $y \in \Omega$,

- either there is $x \in\{\Phi=\bar{\varphi}\} \cap \Omega$ with $|y-x| \leq K(\sigma+\varsigma)^{\beta}$;

- or $y$ belongs to the convex hull of the set $\left\{\bar{\varphi}>h-K(\sigma+\varsigma)^{\beta}\right\}$.

To prove this, we define

$$
\Omega_{\beta}:=\left\{y \in \Omega: \operatorname{dist}(y, \partial \Omega) \geq(\sigma+\varsigma)^{\beta}\right\} .
$$

Of course, with a suitable value of $K$, it suffices to consider the case when $y \in \Omega_{\beta}$. So, let us fix $y \in \Omega_{\beta}$.

Since $\Omega$ is a convex set comparable to a ball of unit size (see (3.8)) and $\Phi$ is a nonnegative concave function bounded by 3 inside $\Omega$, there exists a dimensional constant $C^{\prime}$ such that, for every linear function $L \geq \Phi$ satisfying $L(y)=\Phi(y)$, we have

$$
|\nabla L| \leq \frac{C^{\prime}}{(\sigma+\varsigma)^{\beta}}
$$

By [FJ, Step 4-c], there are $m \leq n$ points $y_{1}, \ldots, y_{m} \in F$ such that $y \in S:=\operatorname{co}\left(\left\{y_{1}, \ldots, y_{m}\right\}\right)$, and all $y_{j}$ 's are contact points:

$$
\Phi\left(y_{j}\right)=L\left(y_{j}\right)=\bar{\varphi}\left(y_{j}\right), \quad j=1, \ldots, m .
$$

If the diameter of $S$ is less than $K(\sigma+\varsigma)^{\beta}$, then its vertices are contact points within $K(\sigma+\varsigma)^{\beta}$ of $y$ and we are done.

Hence, let us assume that the diameter of $S$ is at least $K(\sigma+\varsigma)^{\beta}$. We claim that

$$
\bar{\varphi}\left(y_{i}\right)>h-K \sigma^{\beta} \quad \forall i=1, \ldots, m .
$$

Observe that, if we can prove (5.11), then

$$
y \in S \subset \operatorname{co}\left(\left\{\bar{\varphi}>h-K(\sigma+\varsigma)^{\beta}\right\}\right),
$$

and we are done again.

It remains only to prove (5.11). To begin the proof, given $i \in\{1, \ldots, m\}$, take $j \in\{1, \ldots, m\}$ such that $\left|y_{i}-y_{j}\right| \geq K(\sigma+\varsigma)^{\beta} / 2$ (such a $j$ always exists because of the assumption on the diameter of $S$ ). We rename $i=1$ and $j=2$.

Fix $N \in \mathbb{N}$ to be chosen later. For $x, y \in \Omega$, define

$$
H_{N}(x, y):=\bigcap_{k=0}^{N} \bigcap_{j=0}^{k}\left(\frac{1}{\left(t^{\prime}\right)^{j}\left(t^{\prime \prime}\right)^{k-j}} F[y]-\left(\frac{1}{\left(t^{\prime}\right)^{j}\left(t^{\prime \prime}\right)^{k-j}}-1\right) x\right),
$$

where

$$
F[y]:=F \cap \frac{F-t^{\prime} y}{1-t^{\prime}} \cap \frac{F-t^{\prime \prime} y}{1-t^{\prime \prime}} .
$$


Observe that, since $\Omega$ is convex and by (3.7),

$$
\begin{gathered}
\mathcal{H}^{n-1}(\Omega \backslash F[y]) \leq C \mathcal{H}^{n-1}(\Omega \backslash F) \leq C \varsigma, \\
\mathcal{H}^{n-1}\left(\Omega \backslash\left(\frac{1}{\left(t^{\prime}\right)^{j}\left(t^{\prime \prime}\right)^{k-j}} F[y]-\left(\frac{1}{\left(t^{\prime}\right)^{j}\left(t^{\prime \prime}\right)^{k-j}}-1\right) x\right)\right) \\
=\frac{1}{\left(t^{\prime}\right)^{j(n-1)}\left(t^{\prime \prime}\right)^{(k-j)(n-1)}} \mathcal{H}^{n-1}\left(\left(\left(t^{\prime}\right)^{j}\left(t^{\prime \prime}\right)^{k-j} \Omega+\left(1-\left(t^{\prime}\right)^{j}\left(t^{\prime \prime}\right)^{k-j}\right) x\right) \backslash F[y]\right) \\
\leq \frac{1}{\left(t^{\prime}\right)^{j(n-1)}\left(t^{\prime \prime}\right)^{(k-j)(n-1)}} \mathcal{H}^{n-1}(\Omega \backslash F[y]) \leq \frac{C}{\left(t^{\prime}\right)^{j(n-1)}\left(t^{\prime \prime}\right)^{(k-j)(n-1)}} \varsigma,
\end{gathered}
$$

(and analogously for $t^{\prime \prime}$ ), so

$$
\begin{aligned}
\mathcal{H}^{n-1}\left(\Omega \backslash H_{N}(x, y)\right) & \leq C \sum_{k=0}^{N} \sum_{j=0}^{k} \frac{1}{\left(t^{\prime}\right)^{j(n-1)}\left(t^{\prime \prime}\right)^{(k-j)(n-1)}} \varsigma \\
& \leq C\left(\frac{1}{t^{\prime \prime}}\right)^{N(n-1)} \varsigma,
\end{aligned}
$$

where we used that $t^{\prime \prime} \leq t^{\prime}$. Choose $N$ such that

$$
\left(\frac{\tau}{2}\right)^{N(n-1)}=C \varsigma
$$

for some large dimensional constant $C$. In this way, from (3.8) and (5.12) we get

$$
\mathcal{H}^{n-1}\left(H_{N}\left(y_{2}, y_{1}\right)\right) \geq c_{n} / 2>0
$$

and hence $H_{N}\left(y_{2}, y_{1}\right)$ is nonempty.

Now, choose $w_{0} \in H_{N}\left(y_{2}, y_{1}\right)$, and apply (5.6) iteratively in the following way: if we set $w_{1}^{\prime}:=t^{\prime} w_{0}+\left(1-t^{\prime}\right) y_{2}, w_{1}^{\prime \prime}:=t^{\prime \prime} w_{0}+\left(1-t^{\prime \prime}\right) y_{2}$, then the fact that $w_{0} \in H_{N}\left(y_{2}, y_{1}\right)$ implies that $w_{1}^{\prime}, w_{1}^{\prime \prime} \in F$ (and also that $\left.t^{\prime} y_{1}+\left(1-t^{\prime}\right) w_{0}, t^{\prime \prime} y_{1}+\left(1-t^{\prime \prime}\right) w_{0} \in F\right)$. Hence we can apply (5.6) to obtain

$$
\varphi\left(w_{1}^{\prime}\right)+\varphi\left(w_{1}^{\prime \prime}\right) \geq \varphi\left(y_{2}\right)+\varphi\left(w_{0}\right)-\sigma .
$$

Then define $w_{1}$ to be equal either to $w_{1}^{\prime}$ or to $w_{1}^{\prime \prime}$ so that $\varphi\left(w_{1}\right)=\max \left\{\varphi\left(w_{1}^{\prime}\right), \varphi\left(w_{1}^{\prime \prime}\right)\right\}$. Then, it follows from the equation above that

$$
\varphi\left(w_{1}\right) \geq \frac{\varphi\left(y_{2}\right)+\varphi\left(w_{0}\right)}{2}-\frac{\sigma}{2} .
$$

We now set $w_{2}^{\prime}:=t^{\prime} w_{1}+\left(1-t^{\prime}\right) y_{2}, w_{2}^{\prime \prime}:=t^{\prime \prime} w_{1}+\left(1-t^{\prime \prime}\right) y_{2} \in F$, and apply (5.6) again to get

$$
\varphi\left(w_{2}^{\prime}\right)+\varphi\left(w_{2}^{\prime \prime}\right) \geq \varphi\left(y_{2}\right)+\varphi\left(w_{1}\right)-\sigma \geq(1-1 / 4) \varphi\left(y_{2}\right)+\varphi\left(w_{0}\right) / 4-(1+1 / 2) \sigma .
$$


Again we choose $w_{2} \in\left\{w_{2}^{\prime}, w_{2}^{\prime \prime}\right\}$ so that $\varphi\left(w_{2}\right)=\max \left\{\varphi\left(w_{2}^{\prime}\right), \varphi\left(w_{2}^{\prime \prime}\right)\right\}$ and we keep iterating this construction, so that in $N$ steps we get (recall that $0 \leq \varphi \leq 3$ )

$$
\begin{aligned}
\varphi\left(w_{N}\right) & \geq\left(1-2^{-N}\right) \varphi\left(y_{2}\right)+2^{-N} \varphi\left(w_{0}\right)-2 \sigma \\
& \geq\left(1-2^{-N}\right) \varphi\left(y_{2}\right)-2 \sigma \\
& \geq \varphi\left(y_{2}\right)-3 \cdot 2^{-N}-2 \sigma
\end{aligned}
$$

Hence,

$$
\bar{\varphi}\left(w_{N}\right) \geq \bar{\varphi}\left(y_{2}\right)-3 \cdot 2^{-N}-2 \sigma .
$$

In addition, since $w_{0} \in H_{N}\left(y_{1}, y_{2}\right)$,

$$
y^{\prime}:=t^{\prime} y_{1}+t^{\prime \prime} w_{N} \in F, \quad y^{\prime \prime}:=t^{\prime \prime} y_{1}+t^{\prime} w_{N} \in F .
$$

Since the diameter of $F$ is bounded (see (3.7) and (3.8)) it is easy to check that

$$
\left|w_{N}-y_{2}\right| \leq C\left(t^{\prime}\right)^{N} \leq C(1-\tau / 2)^{N} .
$$

Therefore, by (5.10) we have

$$
\left|L\left(y^{\prime}+y^{\prime \prime}\right)-L\left(y_{1}+y_{2}\right)\right|=\left|L\left(w_{N}-y_{2}\right)\right| \leq C(\sigma+\varsigma)^{-\beta}(1-\tau / 2)^{N} .
$$

Hence, since $y_{1}$ and $y_{2}$ are contact points and $L \geq \bar{\varphi}$, using (5.14) and (5.13) we get

$$
\begin{aligned}
\bar{\varphi}\left(y_{1}\right)+\bar{\varphi}\left(w_{N}\right) & \geq \bar{\varphi}\left(y_{1}\right)+\bar{\varphi}\left(y_{2}\right)-3 \cdot 2^{-N}-2 \sigma \\
& =L\left(y_{1}+y_{2}\right)-3 \cdot 2^{-N}-2 \sigma \\
& \geq L\left(y^{\prime}+y^{\prime \prime}\right)-C\left(2^{-N}+\sigma+(\sigma+\varsigma)^{-\beta}(1-\tau / 2)^{N}\right) \\
& \geq \bar{\varphi}\left(y^{\prime}\right)+\bar{\varphi}\left(y^{\prime \prime}\right)-C(\sigma+\varsigma)^{\min \{\theta, \kappa-\beta\}},
\end{aligned}
$$

where (recall (4.5D)

$$
\theta:=\frac{1}{n-1} \frac{\log (2)}{|\log (\tau / 2)|}, \quad \kappa:=\frac{1}{n-1} \frac{|\log (1-\tau / 2)|}{|\log (\tau / 2)|} .
$$

Now assume by way of contradiction that

$$
\bar{\varphi}\left(y_{1}\right) \leq h-K(\sigma+\varsigma)^{\beta} .
$$

We also have (recalling that $t^{\prime \prime}=1-t^{\prime}$ )

$$
\begin{aligned}
L\left(w_{N}\right) & \leq L\left(w_{N}-y_{2}\right)+L\left(y_{2}\right)=L\left(w_{N}-y_{2}\right)+\bar{\varphi}\left(y_{2}\right) \\
& \leq C(\sigma+\varsigma)^{-\beta}(1-\tau / 2)^{N}+h \leq h+C(\sigma+\varsigma)^{\kappa-\beta}
\end{aligned}
$$

Hence, since $L\left(y_{1}\right)=\bar{\varphi}\left(y_{1}\right)$,

$$
\begin{aligned}
L\left(y^{\prime \prime}\right) & =t^{\prime \prime} \bar{\varphi}\left(y_{1}\right)+t^{\prime} L\left(w_{N}\right) \\
& \leq t^{\prime \prime} h-\frac{K}{2}(\sigma+\varsigma)^{\beta}+t^{\prime} h+C(\sigma+\varsigma)^{\kappa-\beta}<h,
\end{aligned}
$$


provided $K>2 C$ and $\beta \leq \kappa / 2$. Similarly (and more easily since $t^{\prime \prime} \leq t^{\prime}$ ), we have $L\left(y^{\prime}\right)<h$. Since $L \geq \bar{\varphi}$, we have $\max \left\{\bar{\varphi}\left(y^{\prime}\right), \bar{\varphi}\left(y^{\prime \prime}\right)\right\}<h$. Applying (5.7) with $y_{2}$ replaced by $w_{N}$ we get

$$
\bar{\varphi}\left(y^{\prime}\right)+\bar{\varphi}\left(y^{\prime \prime}\right)=\varphi\left(y^{\prime}\right)+\varphi\left(y^{\prime \prime}\right) \geq \varphi\left(y_{1}\right)+\varphi\left(w_{N}\right)+\tau(\sigma+\varsigma)^{\beta}\left|y_{1}-w_{N}\right|^{2},
$$

and since $\left|y_{1}-w_{N}\right| \geq\left|y_{1}-y_{2}\right| / 2 \geq K(\sigma+\varsigma)^{\beta} / 4$ this implies

$$
\bar{\varphi}\left(y^{\prime}\right)+\bar{\varphi}\left(y^{\prime \prime}\right) \geq \bar{\varphi}\left(y_{1}\right)+\bar{\varphi}\left(w_{N}\right)+\frac{\tau K^{2}}{16}(\sigma+\varsigma)^{3 \beta},
$$

which contradicts (5.15) provided we choose $\beta:=\min \left\{\frac{\theta}{3}, \frac{\kappa}{4}\right\}$ and $K$ sufficiently large.

Recalling the definition of $\theta$ and $\kappa$ (see (5.16) $)$, this concludes the proof with the choice

$$
\beta:=\frac{1}{(n-1)|\log (\tau / 2)|} \min \left\{\frac{\log (2)}{3}, \frac{|\log (1-\tau / 2)|}{4}\right\} \geq \frac{\tau}{8(n-1)|\log \tau|} .
$$

Step d: Most of the level sets of $\bar{\varphi}+20(\sigma+\varsigma)^{\beta}|y|^{2}$ are close to their convex hull

This will follow from the fact that it is true for $\psi$. Indeed, define

$$
\bar{\psi}(y):=\min \left\{\bar{\varphi}(y)+20(\sigma+\varsigma)^{\beta}|y|^{2}, h\right\}
$$

Then

$$
\{y \in F: \bar{\psi}(y)>s\}=\left\{\begin{array}{cl}
\{y \in F: \psi(y)>s-2\} & \text { if } s<h \\
\emptyset & \text { if } s \geq h .
\end{array}\right.
$$

Define

$$
H_{1}:=\{s \in \mathbb{R}: s-2 \in H\} \cup[h, \infty), \quad H_{2}:=\mathbb{R} \backslash H_{1} .
$$

Then it follows from (3.10) and (3.7) that

$$
\int_{H_{1}} \mathcal{H}^{n-1}(\operatorname{co}(\{\bar{\psi}>s\}) \backslash\{\bar{\psi}>s\}) d s+\int_{H_{2}} \mathcal{H}^{n-1}(\{\bar{\psi}>s\}) d s \leq 3 \mathcal{H}^{n-1}(\Omega \backslash F)+\varsigma \leq C \varsigma .
$$

Notice that, since $\{\bar{\psi}>s\} \supset\{\bar{\varphi}>s\}$, by (5.8) we have $\mathcal{H}^{n-1}(\{\bar{\psi}>s\}) \geq(\sigma+\varsigma)^{\gamma}$ for all $s<h$. So by (5.18)

$$
\mathcal{H}^{1}\left(H_{2}\right) \leq C(\sigma+\varsigma)^{1-\gamma} .
$$

Step e: $\psi$ is $L^{1}$-close to a concave function

Since the sets $\{\bar{\psi}>s\}$ are decreasing in $s$, so are their convex hulls $\operatorname{co}(\{\bar{\psi}>s\})$. Hence, we can define a new function $\xi: \Omega \rightarrow \mathbb{R}$ with convex level sets given by

$$
\{\xi>s\}:=\operatorname{co}(\{\bar{\psi}>s\}) \quad \text { if } s \in H_{1}, \quad\{\xi>s\}:=\bigcap_{s^{\prime} \in H_{1}, s^{\prime}<s} \operatorname{co}\left(\left\{\bar{\psi}>s^{\prime}\right\}\right) \quad \text { if } s \in H_{2},
$$

Recall that $\Phi$ denotes the concave envelope of $\bar{\phi}$, and in particular, $\bar{\psi} \leq \Phi+C(\sigma+\varsigma)^{\beta}$. It follows from the definition of $\xi$ that

$$
0 \leq \bar{\psi} \leq \xi ; \quad \xi \leq \Phi+C(\sigma+\varsigma)^{\beta} .
$$


By (5.18) and (5.19), we see that $\xi$ satisfies

$$
\int_{\Omega}|\xi-\bar{\psi}| \leq \varsigma+C(\sigma+\varsigma)^{1-\gamma}
$$

Also, because of (5.8), we see that

$$
\mathcal{H}^{n-1}(\{\xi>s\}) \geq(\sigma+\varsigma)^{\gamma} \quad \forall 0 \leq s<h .
$$

Recall from Step c that the contact set $\{\Phi=\bar{\varphi}\}$ is $\varepsilon_{1}$-dense in

$$
\Omega \backslash \operatorname{co}\left(\left\{\bar{\varphi}>h-\varepsilon_{1}\right\}\right)
$$

with $\varepsilon_{1}:=K(\sigma+\varsigma)^{\beta}$.

Let

$$
\varepsilon:=\hat{C} \frac{\varepsilon_{1}}{(\sigma+\varsigma)^{\gamma}}=\hat{C}(\sigma+\varsigma)^{\beta-\gamma},
$$

where $\hat{C}$ is a large universal constant (to be chosen).

We claim that, if $s<h-\varepsilon_{1}$,

$$
\{y \in \Omega: \Phi(y)>s\} \subset \varepsilon \text {-neighborhood of }\{y \in \Omega: \xi(y)>s\} .
$$

To prove (5.24), assume by contradiction that there exists $y \in\{\Phi>s\}$ such that $B_{\varepsilon}(y) \cap\{\xi>$ $s\}=\emptyset$. Since, $s+\varepsilon_{1}<h,(\underline{5.22})$ implies

$$
\mathcal{H}^{n-1}\left(\left\{\xi>s+\varepsilon_{1}\right\}\right) \geq(\sigma+\varsigma)^{\gamma} .
$$

In addition, $\left\{\xi>s+\varepsilon_{1}\right\}$ is a universally bounded convex set, so there is $y^{\prime} \in \Omega$ and $\rho=c(\sigma+\varsigma)^{\gamma}$, with $c>0$ a dimensional constant, such that

$$
B_{\rho}\left(y^{\prime}\right) \subset\left\{\xi>s+\varepsilon_{1}\right\} \subset\{\Phi>s\} .
$$

Since $\Omega$ is universally bounded, there exists $y^{\prime \prime} \in \Omega$ and $r=c \rho \varepsilon$, with $c>0$ a dimensional constant, such that

$$
B_{r}\left(y^{\prime \prime}\right) \subset \operatorname{co}\left(B_{\rho}\left(y^{\prime}\right) \cup\{y\}\right) \cap B_{\varepsilon}(y) \subset\{\Phi>s\} \cap\{\xi \leq s\} .
$$

Thus for any $z \in B_{r}\left(y^{\prime \prime}\right)$,

$$
\bar{\varphi}(z) \leq \xi(z) \leq s<\Phi(z),
$$

and there are no contact points of $\{\Phi=\bar{\varphi}\}$ in $B_{r}\left(y^{\prime \prime}\right)$. But note that for our choice of $\varepsilon, r=c \rho \varepsilon>\varepsilon_{1}$ provided $\hat{C}$ is sufficiently large. This contradicts the $\varepsilon_{1}$-density property, proving (5.24).

Since all level sets of $\xi$ are (universally) bounded convex sets, as a consequence of (5.24) we deduce that

$$
\mathcal{H}^{n-1}(\{\Phi>s\}) \leq \mathcal{H}^{n-1}(\{\xi>s\})+C \varepsilon \quad \forall s<h-\varepsilon_{1} .
$$

Furthermore, since $\xi \leq \Phi+\varepsilon_{1}$, we obviously have that $|\Phi-\xi| \leq 2 \varepsilon_{1}$ on the set $\left\{\xi>h-\varepsilon_{1}\right\}$. Hence, by Fubini's Theorem (and $\varepsilon>\varepsilon_{1}$ ),

$$
\int_{\Omega}|\Phi-\xi| \leq C \varepsilon
$$


Combining this estimate with (5.21) and the fact that $|\bar{\psi}-\bar{\varphi}| \leq C(\sigma+\varsigma)^{\beta}<\varepsilon$, we get

$$
\int_{\Omega}|\Phi-\bar{\varphi}| \leq C \varepsilon
$$

By construction $|\varphi(y)-2-\psi(y)| \leq C(\sigma+\varsigma)^{\beta}$ on $F$ (see (5.5) ). Hence, by (5.9) we have

$$
\begin{aligned}
\int_{\Omega}|\bar{\varphi}(y)-2-\psi(y)| d y & \leq 3|\Omega \backslash F|+\int_{F}[|\bar{\varphi}(y)-\varphi(y)|+|\varphi(y)-2 M-f(y)|] d y \\
& \leq 3\left((\sigma+\varsigma)^{\gamma}+\varsigma\right)+\varepsilon|F| \leq C\left((\sigma+\varsigma)^{\gamma}+\varepsilon\right) .
\end{aligned}
$$

Taking $\gamma=\beta / 2$ and recalling (5.23) and (5.17), this proves (3.11) with $\Psi:=\Phi-2$.

\subsection{A linearity result}

The aim of this section it to show that, if a one dimensional function satisfies on a large set the concavity-type estimate in Remark 4.1 (see Section 4.1) both from above and from below, then it is universally close to a linear function.

Lemma 5.1. Let $0<\tau \leq 1 / 2$ and fix $t^{\prime}$ such that $1 / 2 \leq t^{\prime} \leq 1-\tau / 2$. Let $t^{\prime \prime}=1-t^{\prime}$, and for all $m_{1}, m_{2} \in \mathbb{R}$ define

$$
m_{12}^{\prime}:=t^{\prime} m_{1}+t^{\prime \prime} m_{2} ; \quad m_{12}^{\prime \prime}:=t^{\prime \prime} m_{1}+t^{\prime} m_{2} .
$$

Let $E \subset \mathbb{R}$, and let $f: E \rightarrow \mathbb{R}$ be a bounded measurable function such that

$$
\left|f\left(m_{1}\right)+f\left(m_{2}\right)-f\left(m_{12}^{\prime}\right)-f\left(m_{12}^{\prime \prime}\right)\right| \leq 1 \quad \forall m_{1}, m_{2}, m_{12}^{\prime}, m_{12}^{\prime \prime} \in E .
$$

Assume that there exist points $\bar{m}_{1}, \bar{m}_{2} \in \mathbb{R}$ such that $\bar{m}_{1}, \bar{m}_{2}, \bar{m}_{12}^{\prime}, \bar{m}_{12}^{\prime \prime} \in E$ and $\left|E \cap\left[\bar{m}_{1}, \bar{m}_{2}\right]\right| \geq$ $(1-\varepsilon)\left|\bar{m}_{2}-\bar{m}_{1}\right|$. Then the following hold provided $\varepsilon$ is sufficiently small (the smallness being universal):

(i) There exist a linear function $\ell:\left[\bar{m}_{1}, \bar{m}_{2}\right] \rightarrow \mathbb{R}$ and a universal constant $\bar{M}$, such that

$$
|f-\ell| \leq \bar{M} \quad \text { in } E \cap\left[\bar{m}_{1}, \bar{m}_{2}\right] .
$$

(ii) If in addition $\left|f\left(\bar{m}_{1}\right)\right|+\left|f\left(\bar{m}_{2}\right)\right| \leq K$ for some constant $K$, then $|f| \leq K+\bar{M}$ inside $E$.

Proof. Without loss of generality, we can assume that $\left[\bar{m}_{1}, \bar{m}_{2}\right]=[-1,1]$ and $E \subset[-1,1]$. Given numbers $a \in \mathbb{R}$ and $b>0$, we write $a=O(b)$ if $|a| \leq C b$ for some universal constant $C$.

To prove (i), let us define

$$
\ell(m):=\frac{f(1)-f(-1)}{2} m+\frac{f(1)+f(-1)}{2},
$$

and set $F:=f-\ell$. Observe that $F(-1)=F(1)=0$, and $F$ still satisfies (5.25). Hence, since by assumption $-1,1,1-2 t^{\prime}, 1-2 t^{\prime \prime} \in E$, by (5.25) we get $\left|F\left(1-2 t^{\prime}\right)+F\left(1-2 t^{\prime \prime}\right)\right| \leq 1$. Let us extend $F$ to the whole interval $[-1,1]$ as $F(m)=0$ if $m \notin E$, and set

$$
\bar{M}:=\sup _{m \in[-1,1]}|F(m)| .
$$


We want to show that $\bar{M}$ is universally bounded.

Averaging (5.25) (applied to $F$ in place of $f$ ) with respect to $m_{2} \in E$ and using that $\mid E \cap$ $[-1,1] \mid \geq 2(1-\varepsilon)$, we easily obtain the following bound:

$$
\begin{aligned}
F\left(m_{1}\right)= & \frac{1}{2-2 t^{\prime}} \int_{t^{\prime} m_{1}-\left(1-t^{\prime}\right)}^{t^{\prime} m_{1}+\left(1-t^{\prime}\right)} F(m) d m+\frac{1}{2-2 t^{\prime \prime}} \int_{t^{\prime \prime} m_{1}-\left(1-t^{\prime \prime}\right)}^{t^{\prime \prime} m_{1}+\left(1-t^{\prime \prime}\right)} F(m) d m \\
& -\frac{1}{2} \int_{-1}^{1} F(m) d m+O(1)+O(\varepsilon \bar{M}),
\end{aligned}
$$

from which it follows that

$$
\left|F\left(m^{\prime}\right)-F\left(m^{\prime \prime}\right)\right| \leq C\left(\bar{M}\left|m^{\prime}-m^{\prime \prime}\right|+1+\varepsilon \bar{M}\right) \quad \forall m^{\prime}, m^{\prime \prime} \in E .
$$

Now, pick a point $\tilde{m} \in E$ such that

$$
|F(\tilde{m})| \geq \bar{M}-1
$$

With no loss of generality we assume that $F(\tilde{m}) \geq \bar{M}-1$. Since

$$
\bigcup_{\bar{m}_{0} \in[-1,1]}\left\{-t^{\prime \prime}+\left(1-t^{\prime \prime}\right) \bar{m}_{0}\right\}=\left[-1,1-2 t^{\prime \prime}\right], \quad \bigcup_{\bar{m}_{0} \in[-1,1]}\left\{t^{\prime} \bar{m}_{0}+\left(1-t^{\prime}\right)\right\}=\left[1-2 t^{\prime \prime}, 1\right],
$$

we can find a point $\bar{m}_{0} \in[-1,1]$ such that

$$
\text { either } \quad \tilde{m}=-t^{\prime \prime}+\left(1-t^{\prime \prime}\right) \bar{m}_{0} \quad \text { or } \quad \tilde{m}=t^{\prime} \bar{m}_{0}+\left(1-t^{\prime}\right) .
$$

Without loss of generality we can assume that we are in the fist case.

Set $m_{0}:=-1$. We want to find a point $\hat{m}_{0} \in E$ close to $\bar{m}_{0}$ such that

$$
t^{\prime} m_{0}+\left(1-t^{\prime}\right) \hat{m}_{0}, t^{\prime \prime} m_{0}+\left(1-t^{\prime \prime}\right) \hat{m}_{0} \in E .
$$

Define $C_{t}:=2\left(\frac{1}{1-t^{\prime}}+\frac{1}{1-t^{\prime \prime}}\right)$. Then the above inclusions mean

$$
\hat{m}_{0} \in \frac{E-t^{\prime} m_{0}}{1-t^{\prime}} \cap \frac{E-t^{\prime \prime} m_{0}}{1-t^{\prime \prime}},
$$

and since the latter set contains $[-1,1]$ up to a set of measure $C_{t} \varepsilon$, we can find such a point at a distance at most $C_{t} \varepsilon$ from $\bar{m}_{0}$. Notice that in this way we also get $\left|t^{\prime \prime} m_{0}+\left(1-t^{\prime \prime}\right) \hat{m}_{0}-\tilde{m}\right| \leq C_{t} \varepsilon$, so, by (5.26) and (5.27),

$$
F\left(t^{\prime \prime} m_{0}+\left(1-t^{\prime \prime}\right) \hat{m}_{0}\right) \geq \bar{M}-1-C\left(1+\left(1+C_{t}\right) \varepsilon \bar{M}\right) .
$$

Then, thanks to (5.28), we can apply (5.25) with $F$ in place of $f, m_{1}=m_{0}$, and $m_{2}=\bar{m}_{0}$, to deduce that (recall that $F\left(m_{0}\right)=F(-1)=0$ and that $|F| \leq \bar{M}$ )

$$
\begin{aligned}
F\left(t^{\prime} m_{0}+\left(1-t^{\prime}\right) \hat{m}_{0}\right) & \leq 1+F\left(m_{0}\right)-F\left(t^{\prime \prime} m_{0}+\left(1-t^{\prime \prime}\right) \hat{m}_{0}\right)+F\left(\bar{m}_{0}\right) \\
& \leq 1+(1-\bar{M})+C\left(1+\left(1+C_{t}\right) \varepsilon \bar{M}\right)+\bar{M} \\
& =2+C\left(1+\left(1+C_{t}\right) \varepsilon \bar{M}\right) .
\end{aligned}
$$


We now define $m_{1}:=t^{\prime} m_{0}+\left(1-t^{\prime}\right) \hat{m}_{0}$ and we choose $\bar{m}_{1} \in[-1,1]$ such that

$$
\tilde{m}=t^{\prime \prime} m_{1}+\left(1-t^{\prime \prime}\right) \bar{m}_{1} .
$$

Again we pick a point $\hat{m}_{1} \in\left[\bar{m}_{1}-C_{t} \varepsilon, \bar{m}_{1}+C_{t} \varepsilon\right] \cap E$ such that

$$
t m_{1}^{\prime}+\left(1-t^{\prime}\right) \hat{m}_{1}, t^{\prime \prime} m_{1}+\left(1-t^{\prime \prime}\right) \hat{m}_{1} \in E,
$$

and applying again (5.26) and (5.25) we get

$$
F\left(t^{\prime \prime} m_{1}+\left(1-t^{\prime \prime}\right) \hat{m}_{1}\right) \geq \bar{M}-1-\bar{C}\left(1+\left(1+C_{t}\right) \varepsilon \bar{M}\right),
$$

hence

$$
\begin{aligned}
F\left(t^{\prime} m_{1}+\left(1-t^{\prime}\right) \hat{m}_{1}\right) & \leq 1+F\left(m_{1}\right)-F\left(t^{\prime \prime} m_{1}+\left(1-t^{\prime \prime}\right) \hat{m}_{1}\right)+F\left(\bar{m}_{1}\right) \\
& \leq 1+2+\bar{C}\left(1+\left(1+C_{t}\right) \varepsilon \bar{M}\right)+(1-\bar{M})+\bar{C}\left(1+\left(1+C_{t}\right) \varepsilon \bar{M}\right)+\bar{M} \\
& \leq 4+2 C\left(1+\left(1+C_{t}\right) \varepsilon \bar{M}\right) .
\end{aligned}
$$

Iterating this procedure, after $k$ steps we get

$$
F\left(t^{\prime} m_{k}+\left(1-t^{\prime}\right) \hat{m}_{k}\right) \leq 2(k+1)+(k+1) \bar{C}\left(1+\left(1+C_{t}\right) \varepsilon \bar{M}\right),
$$

and it is easy to check that the points $m_{k}$ and $\bar{m}_{k}$ converge geometrically to $\tilde{m}$ up to an additive error $C_{t} \varepsilon$ at every step, that is

$$
\left|m_{k}-\tilde{m}\right|+\left|\bar{m}_{k}-\tilde{m}\right| \leq \bar{C}\left(2^{-k}+k C_{t} \varepsilon\right) .
$$

Hence, thanks to (5.26) applied with $m^{\prime}=t^{\prime} m_{k}+\left(1-t^{\prime}\right) \hat{m}_{k}$ and $m^{\prime \prime}=\tilde{m}$ we get

$$
\begin{aligned}
\bar{M}-1-2(k+1)\left(2+\bar{C}\left(1+\left(1+C_{t}\right) \varepsilon \bar{M}\right)\right) & \leq F(\tilde{m})-F\left(t^{\prime} m_{k}+\left(1-t^{\prime}\right) \bar{m}_{k}\right) \\
& \leq \bar{C}\left(\bar{M}\left(2^{-k}+k C_{t} \varepsilon\right)+\varepsilon \bar{M}\right)
\end{aligned}
$$

for some universal constant $\bar{C}$. Hence, by choosing $k=N$ a large universal constant so that $\bar{C} 2^{-N} \leq 1 / 2$, we obtain

$$
\bar{M} \leq \frac{\bar{M}}{2}+C(1+N+\varepsilon N \bar{M})
$$

which proves that $\bar{M}$ is universally bounded provided $\varepsilon$ is sufficiently small (the smallness being universal). This proves (i).

To prove (ii), it suffices to observe that if $|f(-1)|+|f(1)| \leq K$ then $|\ell| \leq K$, so (i) gives

$$
|f| \leq|\ell|+|F| \leq K+\bar{M} \text {. }
$$




\subsection{Proof of Proposition 3.4}

After translating and replacing $R$ by $2 R$, we can assume that the barycenter of both $K_{A}$ and $K_{B}$ coincide with the origin. Then observe that

$$
\begin{aligned}
\left\|\chi_{t A} * \chi_{(1-t) B}-\chi_{t K_{A}} * \chi_{(1-t) K_{B}}\right\|_{L^{\infty}\left(\mathbb{R}^{n}\right)} & \leq\left\|\chi_{t A}-\chi_{t K_{A}}\right\|_{L^{1}\left(\mathbb{R}^{n}\right)}+\left\|\chi_{(1-t) B}-\chi_{(1-t) K_{B}}\right\|_{L^{1}\left(\mathbb{R}^{n}\right)} \\
& \leq\left|A \Delta K_{A}\right|+\left|B \Delta K_{B}\right| \leq \zeta .
\end{aligned}
$$

We claim that

$$
\chi_{t K_{A}} * \chi_{(1-t) K_{B}}(x)>\zeta \quad \forall x \in\left(1-C \zeta^{1 / n}\right)\left[t K_{A}+(1-t) K_{B}\right],
$$

for $C$ a universal constant. Indeed, by John's lemma, since $K_{A}$ and $K_{B}$ are convex sets in $B_{R}$ of volume comparable to 1 with barycenter 0 , there is a ball $B_{c}$ centered at 0 such that

$$
B_{c} \subset K_{A} \cap K_{B}, \quad c \geq c_{n} R^{1-n} .
$$

Since we are assuming $R \leq \tau^{-N_{n}}, c$ is a universal positive constant. Let $x=t x_{1}+(1-t) x_{2}$ for some $x_{1} \in\left(1-\delta_{1}\right) K_{A}$ and $x_{2} \in\left(1-\delta_{1}\right) K_{B}$, then

$$
\delta_{1} B_{c}+x_{1} \subset K_{A}, \quad \delta_{1} B_{c}+x_{2} \subset K_{B}
$$

Hence $\delta_{1} B_{c} \subset\left(x_{1}-K_{A}\right)$ and $\delta_{1} B_{c} \subset\left(K_{B}-x_{2}\right)$, and consequently

$$
\tau \delta_{1} B_{c} \subset\left[t\left(x_{1}-K_{A}\right)\right] \cap\left[(1-t)\left(K_{B}-x_{2}\right)\right] .
$$

Thus

$$
\chi_{t K_{A}} * \chi_{(1-t) K_{B}}(x)=\left|\left(x-t K_{A}\right) \cap(1-t) K_{B}\right|=\left|t\left(x_{1}-K_{A}\right) \cap(1-t)\left(K_{B}-x_{2}\right)\right| \geq\left|\tau \delta_{1} B_{c}\right|>\zeta
$$

provided $\delta_{1}=C \zeta^{1 / n}$ for some universal constant $C$, proving the claim.

It follows from (5.29) that $\chi_{t A} * \chi_{(1-t) B}(x)>0$, which implies $x \in S$. In all, we have

$$
\left(1-C \zeta^{1 / n}\right)\left[t K_{A}+(1-t) K_{B}\right] \subset S .
$$

Therefore by (1.3) (since, by assumption, $\delta \leq \zeta$ )

$$
\left|t K_{A}+(1-t) K_{B}\right| \leq\left(1+C \zeta^{1 / n}\right)|S| \leq 1+C \zeta^{1 / n} .
$$

Since

$$
|| K_{A}|-1|+|| K_{B}|-1| \leq C \zeta
$$

(by (1.3) and (3.2)), it follows from Theorem 1.2 that

$$
\left|K_{A} \Delta K_{B}\right| \leq C \zeta^{1 / 2 n} .
$$


(Notice that since $K_{A}$ and $K_{B}$ have the same barycenter, there is not need to translate them.) In particular this immediately implies that

$$
|A \Delta B| \leq C \zeta^{1 / 2 n}
$$

Now observe that, by (1.2) and (5.31) we get

$$
\left|\left(1-C \zeta^{1 / n}\right)\left[t K_{A}+(1-t) K_{B}\right]\right| \geq 1-C \zeta^{1 / n},
$$

and hence it follows from (5.30) and (1.3) that

$$
\left|\left(t K_{A}+(1-t) K_{B}\right) \Delta S\right| \leq C \zeta^{1 / n} .
$$

Consider the convex set $\mathcal{K}_{0}:=\operatorname{co}\left(K_{A} \cup K_{B}\right) \supset t K_{A}+(1-t) K_{B}$. By a simple geometric argument using (5.32), we easily deduce that

$$
\mathcal{K}_{0} \subset\left(1+C \zeta^{1 / 2 n^{2}}\right) K_{A}, \quad \mathcal{K}_{0} \subset\left(1+C \zeta^{1 / 2 n^{2}}\right) K_{B}, \quad \mathcal{K}_{0} \subset\left(1+C \zeta^{1 / 2 n^{2}}\right)\left[t K_{A}+(1-t) K_{B}\right],
$$

so, by (3.2) and (5.33) we obtain

$$
\left|A \Delta \mathcal{K}_{0}\right|+\left|B \Delta \mathcal{K}_{0}\right|+\left|S \Delta \mathcal{K}_{0}\right| \leq C \zeta^{1 / 2 n^{2}}
$$

Finally, we claim that

$$
A \subset\left(1+C \zeta^{1 / 2 n^{3}}\right) \mathcal{K}_{0} .
$$

Indeed, following the argument used in the proof of [C1, Lemma 13.3] (see also [FJ, Proof of Theorem 1.2, Step 5]), let $x \in A \backslash \mathcal{K}_{0}$, denote by $x^{\prime} \in \partial \mathcal{K}_{0}$ the closest point in $\mathcal{K}_{0}$ to $x$, set $\rho:=\left|x-x^{\prime}\right|=\operatorname{dist}\left(x, \mathcal{K}_{0}\right)$, and let $v \in \mathbb{S}^{n-1}$ be the unit normal to a supporting hyperplane to $\mathcal{K}_{0}$ at $x^{\prime}$, that is

$$
\left(z-x^{\prime}\right) \cdot v \leq 0 \quad \forall z \in \mathcal{K} .
$$

Let us define $\mathcal{K}_{\rho}:=\left\{z \in \mathcal{K}_{0}:\left(z-x^{\prime}\right) \cdot v \geq-\frac{t}{1-t} \rho\right\}$. Observe that, since $\mathcal{K}_{0}$ is a bounded convex set with volume close to $1,\left|\mathcal{K}_{\rho}\right| \geq c_{n} \tau^{n} \rho^{n}$ for some dimensional constant $c_{n}>0$. Since $x \in A$ we have

$$
S=t A+(1-t) B \supset\left(t x+(1-t)\left[\mathcal{K}_{\rho} \cap B\right]\right) \cup\left(S \cap \mathcal{K}_{0}\right),
$$

and the two sets in the right hand side are disjoint. This implies that (see (5.34))

$$
|S| \geq \tau^{n}\left(\left|\mathcal{K}_{\rho}\right|-\left|\mathcal{K}_{0} \backslash S\right|\right)+\left|S \cap \mathcal{K}_{0}\right| \geq \rho^{n} / C+|S|-C \zeta^{1 / 2 n^{2}}
$$

from which we deduce

$$
\rho \leq C \zeta^{1 / 2 n^{3}}
$$

Since $x$ is arbitrary, this implies that $A$ is contained inside the $\left(C \zeta^{1 / 2 n^{3}}\right)$-neighborhood of $\mathcal{K}_{0}$, proving the claim. 
Since the analogous statement holds for $B$, we obtain that

$$
A \cup B \subset \mathcal{K}:=\left(1+C \zeta^{1 / 2 n^{3}}\right) \mathcal{K}_{0},
$$

and (thanks to (5.34) $)$

$$
\left|\mathcal{K}_{0} \backslash A\right|+\left|\mathcal{K}_{0} \backslash B\right| \leq C \zeta^{1 / 2 n^{3}}
$$

as desired.

\section{References}

[AGS] Ambrosio, L.; Gigli, N.; Savaré, G. Gradient flows in metric spaces and in the space of probability measures. Second edition. Lectures in Mathematics ETH Zürich. Birkhäuser Verlag, Basel, 2008.

[BB1] Ball, K. M.; Böröczky, K. J. Stability of some versions of the Prékopa-Leindler inequality. Monatsh. Math. 163 (2011), no. 1, 1-14.

[BB2] Ball, K. M.; Böröczky, K. J. Stability of the Prékopa-Leindler inequality. Mathematika 56 (2010), no. 2, 339-356.

[BP] Brasco, L.; Pratelli, A. Sharp stability of some spectral inequalities. Geom. Funct. Anal. 22 (2012), no. 1, 107-135.

[C1] Christ M. Near equality in the two-dimensional Brunn-Minkowski inequality. Preprint, 2012. Available online at http://arxiv.org/abs/1206.1965

[C2] Christ M. Near equality in the Brunn-Minkowski inequality. Preprint, 2012. Available online at http://arxiv.org/abs/1207.5062

[C3] Christ M. An approximate inverse Riesz-Sobolev inequality. Preprint, 2012. Available online at http://arxiv.org/abs/1112.3715

[C4] Christ M. Personal communication.

[D] Diskant, V. I. Stability of the solution of a Minkowski equation. (Russian) Sibirsk. Mat. Ž. 14 (1973), 669-673, 696.

[FJ] Figalli A.; Jerison D. Quantitative stability for sumsets in $\mathbb{R}^{n}$. J. Europ. Math. Soc. (JEMS), to appear.

[FMP1] Figalli, A.; Maggi, F.; Pratelli, A. A mass transportation approach to quantitative isoperimetric inequalities. Invent. Math. 182 (2010), no. 1, 167-211.

[FMP2] Figalli, A.; Maggi, F.; Pratelli, A. A refined Brunn-Minkowski inequality for convex sets. Ann. Inst. H. Poincaré Anal. Non Linéaire 26 (2009), no. 6, 2511-2519.

[G] Groemer, H. On the Brunn-Minkowski theorem. Geom. Dedicata 27 (1988), no. 3, 357-371. 
[J] John F. Extremum problems with inequalities as subsidiary conditions. In Studies and Essays Presented to R. Courant on his 60th Birthday, January 8, 1948, pages 187-204. Interscience, New York, 1948.

[S] Schneider, R. Convex bodies: the Brunn-Minkowski theory. Encyclopedia of Mathematics and its Applications, 44. Cambridge University Press, Cambridge, 1993. 